\title{
Birational Weyl group action arising from a nilpotent Poisson algebra
}

\author{
Masatoshi NOUMI and Yasuhiko YAMADA \\ Department of Mathematics, Kobe University \\ Rokko, Kobe 657-8501, Japan
}

\begin{abstract}
We propose a general method to realize an arbitrary Weyl group of Kac-Moody type as a group of birational canonical transformations, by means of a nilpotent Poisson algebra.
\end{abstract}

\section{Introduction}

In this paper we propose a general method to realize an arbitrary Weyl group of Kac-Moody type as a group of birational canonical transformations. Our construction is formulated by means of a nilpotent Poisson algebra. It can be regarded as a conceptual generalization of the birational Weyl group actions proposed in our previous paper [9]. We also discuss a certain cocycle related to this realization and its regularity, and give a proof to a generalization of the regularity conjecture [9].

The plan of this paper is as follows. We give a summary of our main results in Section 1. Fixing a generalized Cartan matrix $A$, we take as a datum a nilpotent Poisson algebra $\mathcal{A}_{0}$ generated by a set of elements $\varphi_{i}(i \in I)$ satisfying the Serre relations (specified by the GCM $A$ ) with respect to the adjoint action by the Poisson bracket. Starting from such an $\mathcal{A}_{0}$, we formulate a method to realize the Weyl group $W=W(A)$ associated with $A$, as a group of birational canonical transformations of a field of rational functions defined by $\mathcal{A}_{0}$ (Theorem 1.1). We also introduce $\tau$-functions in Theorem 1.2 for our realization, and formulate in Theorem 1.3 a certain regularity property of the $\tau$-cocycle arising from the transformations of $\tau$-functions. After describing explicit examples in the cases of rank 2, we give in Section 3 a proof of Theorems 1.1 and 1.2 .

In Section 4, we explain a Lie theoretic background of our birational realization of the Weyl group, in terms of Kac-Moody Lie algebras and Kac-Moody groups. In fact, we consider the birational dressing action of a lift $\dot{W}$ of the Weyl group on the Borel subgroup, induced through the Gauss decomposition in the Kac-Moody group. Our realization is then obtained by transferring this dressing action to the Borel subalgebra through the adjoint action. We give a 
proof of regularity of the $\tau$-cocycle (Theorem 1.3) in Section 5 by using the geometric interpretation of Section 4. Finally in Section 6, we give some remarks related to our birational realization of Weyl groups.

\section{Summary of results}

\subsection{Birational realization of the Weyl group}

Let $A=\left(a_{i j}\right)_{i, j \in I}$ be a generalized Cartan matrix (GCM for short). By definition, $A$ is an integer matrix satisfying the conditions

$$
a_{j j}=2 ; \quad a_{i j} \leq 0 \quad(i \neq j) ; \quad a_{i j}=0 \quad \Longleftrightarrow \quad a_{j i}=0,
$$

for any $i, j \in I$. When the indexing set is infinite, we always assume that $A$ is locally finite; i.e., for any $i \in I, a_{i j}=0$ except for a finite number of $j$ 's. We denote the root lattice for $A$ by $Q=\bigoplus_{i \in I} \mathbb{Z} \alpha_{i}$ and the coroot lattice by $Q^{\vee}=\bigoplus_{i \in I} \mathbb{Z} h_{i}$, where $\alpha_{i}$ and $h_{i}$ are the simple roots and the simple coroots, respectively. The canonical pairing $\langle\rangle:, Q^{\vee} \times Q \rightarrow \mathbb{Z}$ between the two lattices is defined by $\left\langle h_{i}, \alpha_{j}\right\rangle=a_{i j}(i, j \in I)$. The Weyl group $W(A)$ for $A$ is defined by the generators $r_{i}(i \in I)$ with fundamental relations

$$
r_{i}^{2}=1, \quad\left(r_{i} r_{j}\right)^{m_{i j}}=1 \quad(i \neq j),
$$

where $m_{i j}=2,3,4,6$ or $\infty$ according as $a_{i j} a_{j i}=0,1,2,3$, or $\geq 4$. This group acts naturally on $Q$ and $Q^{\vee}$ by

$$
r_{i} . \alpha=\alpha-\alpha_{i}\left\langle h_{i}, \alpha\right\rangle \quad(\alpha \in Q), \quad r_{i} . h=h-\left\langle h, \alpha_{i}\right\rangle h_{i} \quad\left(h \in Q^{\vee}\right),
$$

respectively, so that $\langle w \cdot h, w . \alpha\rangle=\langle h, \alpha\rangle$ for any $h \in Q^{\vee}$ and $\alpha \in Q$. Let $\mathbb{C}[\lambda]$ be the polynomial ring in the indeterminates $\lambda=\left(\lambda_{i}\right)_{i \in I}$. In the following context, each $\lambda_{i}$ will be regarded as a variable corresponding to the simple coroot $h_{i}$. The Weyl group $W(A)=\left\langle r_{i}(i \in I)\right\rangle$ acts on $\mathbb{C}[\lambda]$ as a group of automorphisms such that $r_{j}\left(\lambda_{i}\right)=\lambda_{i}-a_{i j} \lambda_{j}$ for $i, j \in I$.

By a Poisson algebra, we mean a commutative $\mathbb{C}$-algebra $\mathcal{A}$ endowed with a skew-symmetric bilinear form $\{\}:, \mathcal{A} \times \mathcal{A} \rightarrow \mathcal{A}$, called the Poisson bracket, such that

(i) $\{f g, h\}=\{f, h\} g+f\{g, h\}, \quad\{f, g h\}=\{f, g\} h+g\{f, h\}$,

(ii) $\{f,\{g, h\}\}+\{g,\{h, f\}\}+\{h,\{f, g\}\}=0$,

for any $f, g, h \in \mathcal{A}$. A homomorphism $T: \mathcal{A} \rightarrow \mathcal{B}$ between two Poisson algebras will be called a canonical transformation:

$$
T(f g)=T(f) T(g), \quad T(\{f, g\})=\{T(f), T(g)\} \quad(f, g \in \mathcal{A}) .
$$

We now fix a generalized Cartan matrix $A=\left(a_{i j}\right)_{i, j \in I}$, and a Poisson algebra $\mathcal{A}_{0}$. We assume that the algebra $\mathcal{A}_{0}$ has no zerodivisors, and that as a Poisson algebra, $\mathcal{A}_{0}$ is generated by a set of nonzero elements $\left(\varphi_{i}\right)_{i \in I}$ such that

$$
\operatorname{ad}_{\{\}}\left(\varphi_{i}\right)^{-a_{i j}+1}\left(\varphi_{j}\right)=0 \quad(i \neq j)
$$


where $\operatorname{ad}_{\{\}}(f)=\{f, \cdot\}$ stands for the adjoint action by the Poisson bracket. We denote by $\mathcal{A}=\mathcal{A}_{0}[\lambda]$ the ring of polynomials in the $\lambda$-variables with coefficients in $\mathcal{A}_{0}$, and by $\mathcal{K}=Q(\mathcal{A})$ the field of fractions of $\mathcal{A}$. Roughly speaking, $\mathcal{K}$ is the field of rational functions in the variables $\lambda_{j}$, and $\varphi_{j}$ together with the Poisson brackets among $\varphi_{j}$ 's:

$$
\mathcal{K}=\mathbb{C}\left(\lambda_{j} ; \varphi_{j},\left\{\varphi_{i}, \varphi_{j}\right\},\left\{\varphi_{i},\left\{\varphi_{j}, \varphi_{k}\right\}\right\}, \ldots\right) .
$$

Note that the Poisson bracket $\{$,$\} of \mathcal{A}_{0}$ extends uniquely to $\mathcal{K}$ so that $\left\{\lambda_{i}, \varphi\right\}=$ $0(\varphi \in \mathcal{K})$ for any $i \in I$. As to the Weyl group action on the $\lambda$-variables, we use the same notation $r_{i}$ for the $\mathcal{A}_{0}$-linear automorphism of $\mathcal{K}$ defined by $r_{i}\left(\lambda_{j}\right)=\lambda_{j}-a_{j i} \lambda_{i}(j \in I)$. For each $i \in I$, we define a homomorphism $s_{i}: \mathcal{A} \rightarrow \mathcal{A}\left[\varphi_{j}^{-1}(j \in I)\right]$ as the composition

$$
s_{i}=\exp \left(\frac{\lambda_{i}}{\varphi_{i}} \operatorname{ad}_{\{\}}\left(\varphi_{i}\right)\right) \circ r_{i} \quad(i \in I) .
$$

Note that, for any $\psi \in \mathcal{A}_{0}$, the action of $s_{i}$ on $\psi$ is determined as the finite sum

$$
s_{i}(\psi)=\psi+\frac{\lambda_{i}}{\varphi_{i}}\left\{\varphi_{i}, \psi\right\}+\frac{1}{2 !}\left(\frac{\lambda_{i}}{\varphi_{i}}\right)^{2}\left\{\varphi_{i},\left\{\varphi_{i}, \psi\right\}\right\}+\cdots,
$$

since the action of $\operatorname{ad}_{\{\}}\left(\varphi_{i}\right)$ is locally nilpotent on $\mathcal{A}_{0}$. These homomorphisms $s_{i}: \mathcal{A} \rightarrow \mathcal{A}\left[\varphi_{j}^{-1}(j \in I)\right](i \in I)$ extend to automorphisms of $\mathcal{K}=Q(\mathcal{A})$, for which we use the same notation $s_{i}$.

Theorem 1.1 The automorphism $s_{i}(i \in I)$ of the field of fractions $\mathcal{K}=Q(\mathcal{A})$, defined as above, give a realization of the Weyl group $W(A)$ for the GCM A, as a group of canonical transformations of $\mathcal{K}$. Namely,

(1) These automorphisms preserve the Poisson bracket of $\mathcal{K}:$ For each $i \in I$,

$$
s_{i}(\{\varphi, \psi\})=\left\{s_{i}(\varphi), s_{i}(\psi)\right\} \quad(\varphi, \psi \in \mathcal{K}) .
$$

(2) They satisfy the fundamental relations for the generators of $W(A)$ :

$$
s_{i}^{2}=1, \quad\left(s_{i} s_{j}\right)^{m_{i j}}=1 \quad(i \neq j),
$$

where $m_{i j}=2,3,4,6$ or $\infty$ according as $a_{i j} a_{j i}=0,1,2,3$, or $\geq 4$.

Theorem 1.1 is a systematic generalization of the realization of the Weyl group we discussed previously [9], in terms of nilpotent Poisson algebras. (See Remark at the end of this section.)

Our realization of the Weyl group is closely related to the universal exponential solution to the Yang-Baxter equation, due to S. Fomin and A.N. Kirillov [2]. In order to clarify the point, let us define the $R$-operator $R_{i}(t)$ with a formal parameter $t$ by

$$
R_{i}(t)=\exp \left(\frac{t}{\varphi_{i}} \operatorname{ad}_{\{\}}\left(\varphi_{i}\right)\right)
$$


for each $i \in I$, so that $s_{i}=R_{i}\left(\lambda_{i}\right) \circ r_{i}$. The Coxeter relation $\left(s_{i} s_{j}\right)^{m_{i j}}=1$ $(i \neq j)$ is then equivalent to the following Yang-Baxter equation, according to the type of the root system of rank two defined by $\alpha_{i}$ and $\alpha_{j}$ :

$$
\begin{array}{cc}
\left(2 A_{1}\right) & R_{i}(a) R_{j}(b)=R_{j}(b) R_{i}(a), \\
\left(A_{2}\right) & R_{i}(a) R_{j}(a+b) R_{i}(b)=R_{j}(b) R_{i}(a+b) R_{j}(a), \\
\left(B_{2}\right) & R_{i}(a) R_{j}(a+b) R_{i}(a+2 b) R_{j}(b) \\
& =R_{j}(b) R_{i}(a+2 b) R_{j}(a+b) R_{i}(a), \\
\left(G_{2}\right) & R_{i}(a) R_{j}(a+b) R_{i}(2 a+3 b) R_{j}(a+2 b) R_{i}(a+3 b) R_{j}(b) \\
& =R_{j}(b) R_{i}(a+3 b) R_{j}(a+2 b) R_{i}(2 a+3 b) R_{j}(a+b) R_{i}(a),
\end{array}
$$

where $a=\lambda_{i}$ and $b=\lambda_{j}$. These four cases corresponds to the values $\left(a_{i j}, a_{j i}\right)=$ $(0,0),(-1,-1),(-2,-1),(-3,-1)$, respectively.

\section{$1.2 \tau$-Functions and the $\tau$-cocycle}

We now introduce a new set of variables $\tau_{i}(i \in I)$, called $\tau$-functions, and extend the action of the Weyl group to these variables. We denote by $\mathcal{K}\left[\tau^{ \pm 1}\right]=$ $\mathcal{K}\left[\tau_{i}^{ \pm 1}(i \in I)\right]$ the ring of Laurent polynomials in the indeterminates $\tau_{i}(i \in I)$ with coefficients in $\mathcal{K}$.

Theorem 1.2 Extend the automorphisms $s_{i}(i \in I)$ of $\mathcal{K}$ to those of $\mathcal{K}\left[\tau^{ \pm 1}\right]$ by the following action on the $\tau$-functions:

$$
s_{i}\left(\tau_{j}\right)=\tau_{j} \quad(i \neq j), \quad s_{i}\left(\tau_{i}\right)=\varphi_{i} \tau_{i} \prod_{k \in I} \tau_{k}^{-a_{k i}} .
$$

Then these $s_{i}(i \in I)$ again give a realization of the Weyl group $W(A)$ as a group of automorphisms of $\mathcal{K}\left[\tau^{ \pm 1}\right]$.

(For a more intrinsic formulation of this theorem, see Remark at the end of Section 4.)

The action of $s_{i}$ on $\tau$-functions, defined above, is a multiplicative analogue of that of $r_{i}$ on the fundamental weights $\Lambda_{j}$ (modulo null roots), except for the factor $\varphi_{i}$. Let $L=\bigoplus_{i \in I} \mathbb{Z} \Lambda_{i}$ be the free $\mathbb{Z}$-submodule of $\operatorname{Hom}_{\mathbb{Z}}\left(Q^{\vee}, \mathbb{Z}\right)$, generated by the dual basis $\left(\Lambda_{i}\right)_{i \in I}$ of $\left(h_{i}\right)_{i \in I}$. The Weyl group $W(A)=\left\langle r_{i}(i \in I)\right\rangle$ act on $L$ so that

$$
r_{i}\left(\Lambda_{j}\right)=\Lambda_{j} \quad(i \neq j), \quad r_{i}\left(\Lambda_{i}\right)=\Lambda_{i}-\sum_{k \in I} \Lambda_{k} a_{k, i} .
$$

Note that $\langle w \cdot h, w \cdot \Lambda\rangle=\langle h, \Lambda\rangle\left(h \in Q^{\vee}, \Lambda \in L\right)$ for any $w \in W(A)$ and that the natural homomorphism $Q \rightarrow L$ is a $W(A)$-homomorphism.

In what follows we denote by $W=\left\langle s_{i}(i \in I)\right\rangle$ the Weyl group $W(A)$ on the generators $s_{i}(i \in I)$. We introduce the notation of formal exponentials by setting

$$
\tau^{\Lambda}=\prod_{i \in I} \tau_{i}^{\left\langle h_{i}, \Lambda\right\rangle} \quad(\Lambda \in L),
$$


so that $\tau_{i}=\tau^{\Lambda_{i}}$ for $i \in I$. Then the ring $\mathcal{K}\left[\tau^{ \pm 1}\right]$ of Laurent polynomials is isomorphic to the group $\operatorname{ring} \mathcal{K}[L]$. The action of $s_{i}$ on the $\tau$-functions, as defined in Theorem 1.2, is rewritten in the form

$$
s_{i}\left(\tau^{\Lambda}\right)=\varphi_{i}^{\left\langle h_{i}, \Lambda\right\rangle} \tau^{r_{i} \cdot \Lambda} \quad(\Lambda \in L) .
$$

This implies that, for any $w \in W$ and $\Lambda \in L$, there exists a unique element $\phi_{w}(\Lambda) \in \mathcal{K}$ such that

$$
w\left(\tau^{\Lambda}\right)=\phi_{w}(\Lambda) \tau^{w \cdot \Lambda}
$$

Note that these $\phi_{w}(\Lambda)$ are determined by the following cocycle condition:

$$
\begin{aligned}
& \phi_{w}\left(\Lambda+\Lambda^{\prime}\right)=\phi_{w}(\Lambda) \phi_{w}\left(\Lambda^{\prime}\right) \quad\left(\Lambda, \Lambda^{\prime} \in L\right), \\
& \phi_{1}(\Lambda)=1, \quad \phi_{s_{i}}(\Lambda)=\varphi_{i}^{\left\langle h_{i}, \Lambda\right\rangle} \quad(i \in I), \\
& \phi_{w w^{\prime}}(\Lambda)=w\left(\phi_{w^{\prime}}(\Lambda)\right) \phi_{w}\left(w^{\prime} . \Lambda\right) \quad\left(w, w^{\prime} \in W\right) .
\end{aligned}
$$

This family $\phi=\left(\phi_{w}(\Lambda)\right)_{w \in W, \Lambda \in L}$ of elements of $\mathcal{K}$ will be called the $\tau$-cocycle. In fact, the correspondence

$$
\phi: W \rightarrow \operatorname{Hom}\left(L, \mathcal{K}^{\times}\right): w \mapsto\left(\Lambda \mapsto \phi_{w}(\Lambda)\right)
$$

defines a 1-cocycle of the Weyl group $W(A)$ with coefficients in the $W(A)$ bimodule $\operatorname{Hom}\left(L, \mathcal{K}^{\times}\right)$.

The $\tau$-cocycle determines completely the action of the Weyl group on $\mathcal{K}$. Note that, by the definition, the generators $\varphi_{j}$ for the Poisson algebra $\mathcal{A}_{0}$ are expressed as

$$
\varphi_{j}=\frac{\tau_{j} s_{j}\left(\tau_{j}\right)}{\prod_{i \neq j} \tau_{i}^{-a_{i j}}} \quad(j \in I),
$$

multiplicatively in terms of $\tau$-functions. This implies that

$$
w\left(\varphi_{j}\right)=\frac{\phi_{w}\left(\Lambda_{j}\right) \phi_{w s_{j}}\left(\Lambda_{j}\right)}{\prod_{i \neq j} \phi_{w}\left(\Lambda_{i}\right)^{-a_{i j}}}
$$

for any $j \in I, \quad w \in W$.

By the definition of the $\tau$-cocycle, each $\phi_{w}\left(\Lambda_{j}\right)$ is a priori an element of the field of fractions $\mathcal{K}=Q(\mathcal{A})$ of $\mathcal{A}=\mathcal{A}_{0}[\lambda]$. It turns out, however, that the $\tau$-cocycle has a remarkable regularity in the following sense.

Theorem 1.3 Suppose that $A=\left(a_{i j}\right)_{i j \in I}$ is a symmetrizable GCM. Then, for any $w \in W$ and $j \in I$, one has $\phi_{w}\left(\Lambda_{j}\right) \in \mathcal{A}$, namely, $\phi_{w}\left(\Lambda_{j}\right)$ is a polynomial in $\left(\lambda_{i}\right)_{i \in I}$ with coefficients in $\mathcal{A}_{0}$.

Theorem implies that, if $\Lambda \in L$ is dominant, i.e., $\left\langle h_{i}, \Lambda\right\rangle \geq 0$ for any $i \in I$, then one has $\phi_{w}(\Lambda) \in \mathcal{A}=\mathcal{A}_{0}[\lambda]$ for any $w \in W$. When $\overline{\mathcal{A}}_{0}$ has a $\mathbb{Z}$-form in an appropriate sense, one can also show that $\phi_{w}(\Lambda)$ are defined over $\mathbb{Z}$. (See Remark at the end of Section 5.) 
These $\phi_{w}(\Lambda)$ can be regarded as a generalization of the so-called Umemura polynomials for generic solutions of the Painlevé equations ([8]). It would be an interesting problem to investigate combinatorial properties of $\phi_{w}(\Lambda)$.

Remark: Suppose that the generalized Cartan matrix $A$ is symmetrizable, and take nonzero rational numbers $\epsilon_{i}(i \in I)$ such that $a_{i j} \epsilon_{j}=a_{j i} \epsilon_{i}(i, j \in I)$. In this case, the realization of the Weyl group discussed in our previous paper [9] can be recovered essentially from the construction of this section by a special choice of the nilpotent Poisson algebra $\mathcal{A}_{0}$. For $\mathcal{A}_{0}$, take the Poisson algebra, truncated at height 2 , defined by the generators $\varphi_{i}(i \in I)$ with the Serre relations

$$
\operatorname{ad}_{\{\}}\left(\varphi_{i}\right)^{-a_{i j}+1}\left(\varphi_{j}\right)=0 \quad(i \neq j),
$$

and

$$
\left\{\varphi_{i},\left\{\varphi_{j}, \varphi_{k}\right\}\right\}=0 \quad(i, j, k \in I) .
$$

To be consistent with the previous notation [9], set $\alpha_{i}=\lambda_{i} / \epsilon_{i}$ and $u_{i j}=$ $\epsilon_{i}\left\{\varphi_{i}, \varphi_{j}\right\}$. Since $\left\{u_{i j}, \varphi\right\}=0$ for any $\varphi \in \mathcal{A}_{0}$, one can treat $u_{i j}$ as constants.

\section{Examples of rank 2}

In this section we give examples of our realization of the Weyl groups for generalized Cartan matrices of rank 2. In the following examples, we use the notation

$$
\alpha=\alpha_{1}, \quad \beta=\alpha_{2}, \quad a=\lambda_{1}, \quad b=\lambda_{2} .
$$

\subsection{Case of $2 A_{1}$}

We set $x=\varphi_{1}, y=\varphi_{2}$ and $\{x, y\}=0$. In this case, our realization of the Weyl group is trivial on the variables $x, y$.

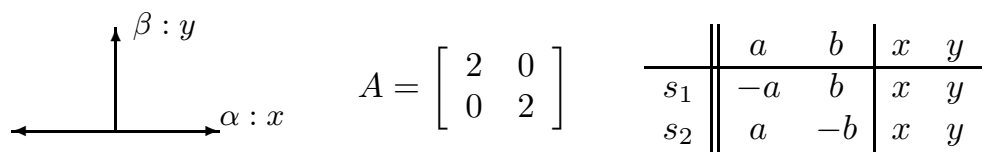

\subsection{Case of $A_{2}$}

We set $x=\varphi_{1}, y=\varphi_{2}$, and $z=\{x, y\}=\left\{\varphi_{1}, \varphi_{2}\right\}$.

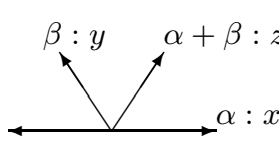

$$
A=\left[\begin{array}{cc}
2 & -1 \\
-1 & 2
\end{array}\right]
$$

\begin{tabular}{c|ccc}
$\{\}$, & $x$ & $y$ & $z$ \\
\hline$x$ & 0 & $z$ & 0 \\
$y$ & $-z$ & 0 & 0 \\
$z$ & 0 & 0 & 0
\end{tabular}

We take the Poisson algebras $\mathcal{A}_{0}=\mathbb{C}[x, y, z]$ (or any quotient Poisson algebra of $\mathbb{C}[x, y, z]$ without zerodivisors), and set $\mathcal{A}=\mathbb{C}[a, b, x, y, z]$. By the table of the 
Poisson bracket indicated above, the action of $s_{1}, s_{2}$ is determined as follows:

\begin{tabular}{c||cc|ccc} 
& $a$ & $b$ & $x$ & $y$ & $z$ \\
\hline$s_{1}$ & $-a$ & $b+a$ & $x$ & $y+\frac{a z}{x}$ & $z$ \\
$s_{2}$ & $a+b$ & $-b$ & $x-\frac{b z}{y}$ & $y$ & $z$
\end{tabular}

The automorphisms $s_{1}, s_{2}$ defined by this table give a realization of the Weyl group $W\left(A_{2}\right)=\left\langle s_{1}, s_{2}\right\rangle \stackrel{\sim}{\rightarrow} \mathfrak{S}_{3}$ as a group of canonical transformations on the field of rational functions $\mathcal{K}=Q(\mathcal{A})=\mathbb{C}(a, b, x, y, z)$.

The $\tau$-cocycle $\phi=\left(\phi_{w}(\Lambda)\right)_{w, \Lambda}$ for this realization is given as follows.

\begin{tabular}{c|cccccc}
$\Lambda \backslash w$ & 1 & $s_{1}$ & $s_{2}$ & $s_{2} s_{1}$ & $s_{1} s_{2}$ & $s_{1} s_{2} s_{1}=s_{2} s_{1} s_{2}$ \\
\hline$\Lambda_{1}$ & 1 & $x$ & 1 & $x y-b z$ & $x$ & $x y-b z$ \\
$\Lambda_{2}$ & 1 & 1 & $y$ & $y$ & $x y+a z$ & $x y+a z$
\end{tabular}

We remark that the value $\phi_{w_{0}}\left(\Lambda_{1}\right)$ of the $\tau$-cocyle at the longest element $w_{0}$ can be determined in two ways as

$$
\phi_{s_{2} s_{1} s_{2}}\left(\Lambda_{1}\right)=\varphi_{2} s_{2}\left(\varphi_{1}\right), \quad \phi_{s_{1} s_{2} s_{1}}\left(\Lambda_{1}\right)=s_{1}\left(\varphi_{2} s_{2}\left(\varphi_{1}\right)\right),
$$

by using the cocycle property for the two reduced decompositions of $w_{0}$. One can verify that $\varphi_{2} s_{2}\left(\varphi_{1}\right)=y\left(x-\frac{b z}{y}\right)=x y-b z$ is invariant with respect to $s_{1}$, which guarantees the equality of the two expressions above.

\subsection{Case of $B_{2}$}

We set $x=\varphi_{1}, y=\varphi_{2}$ and

$$
\begin{aligned}
& z=\{x, y\}=\left\{\varphi_{1}, \varphi_{2}\right\}, \quad w=\frac{1}{2}\{x, z\}=\frac{1}{2}\left\{\varphi_{1},\left\{\varphi_{1}, \varphi_{2}\right\}\right\} . \\
& \beta: y \quad \alpha+\beta: z 2 \alpha+\beta: w \\
& \alpha=x
\end{aligned}
$$

The Poisson bracket and the action of $s_{1}, s_{2}$ are defined by the following tables:

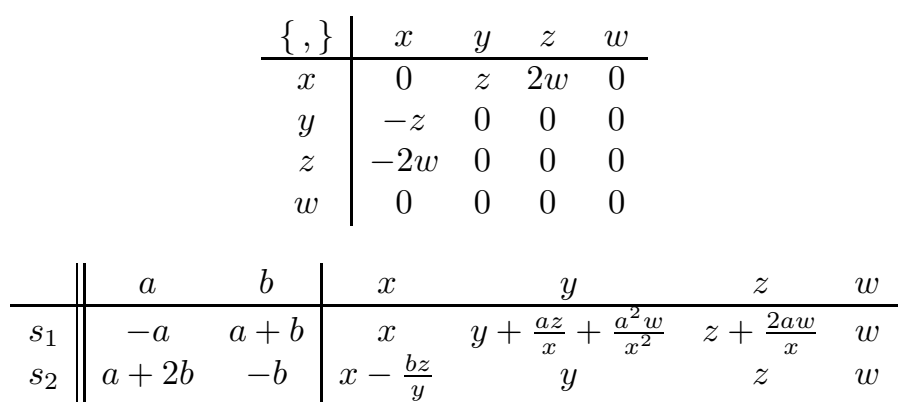


From the nilpotent Poisson algebra $\mathcal{A}_{0}=\mathbb{C}[x, y, z, w]$, we obtain the realization of the Weyl group $W\left(B_{2}\right)$ as a group of canonical transformations on the field of rational functions $\mathcal{K}=\mathbb{C}(a, b, x, y, z, w)$.

The $\tau$-cocycle $\phi=\left(\phi_{w}(\Lambda)\right)_{w, \Lambda}$ for this realization is given as follows.

\begin{tabular}{c|ccccc} 
& 1 & $s_{1}$ & $s_{2}$ & $s_{2} s_{1}$ & $s_{1} s_{2}$ \\
\hline$\Lambda_{1}$ & 1 & $x$ & 1 & $x y-b z$ & $x$ \\
$\Lambda_{2}$ & 1 & 1 & $y$ & $y$ & $x^{2} y+a x z+a^{2} w$
\end{tabular}

\begin{tabular}{c|cc} 
& $s_{1} s_{2} s_{1}$ & $s_{2} s_{1} s_{2}$ \\
\hline$\Lambda_{1}$ & $x^{2} y-b x z-a(a+2 b) w$ & $x y-b z$ \\
$\Lambda_{2}$ & $x^{2} y+a x z+a^{2} w$ & $x^{2} y^{2}+a x y z-(a+b) b z^{2}+(a+2 b)^{2} y w$
\end{tabular}

We remark that

$$
\phi_{s_{1} s_{2} s_{1}}\left(\Lambda_{1}\right)=\varphi_{1} s_{1}\left(\varphi_{2} s_{2}\left(\varphi_{1}\right)\right)=x^{2} y-b x z-a(a+2 b) w
$$

is $s_{2}$-invariant. From this fact, it follows that the two expressions

$$
\phi_{s_{1} s_{2} s_{1} s_{2}}\left(\Lambda_{1}\right)=\phi_{s_{1} s_{2} s_{1}}\left(\Lambda_{1}\right), \quad \phi_{s_{2} s_{1} s_{2} s_{1}}\left(\Lambda_{1}\right)=s_{2}\left(\phi_{s_{1} s_{2} s_{1}}\left(\Lambda_{1}\right)\right)
$$

give the same value $\phi_{w_{0}}\left(\Lambda_{1}\right)$ for the longest element $w_{0}$ of the Weyl group. Similarly, $\phi_{w_{0}}\left(\Lambda_{2}\right)$ is determined consistently from the $s_{1}$-invariance of

$$
\begin{aligned}
\phi_{s_{2} s_{1} s_{2}}\left(\Lambda_{2}\right) & =\varphi_{2} s_{2}\left(\varphi_{1}^{2} s_{1}\left(\varphi_{2}\right)\right) \\
& =x^{2} y^{2}+a x y z-(a+b) b z^{2}+(a+2 b)^{2} y w .
\end{aligned}
$$

\subsection{Case of $G_{2}$}

We set $u=\varphi_{1}, v=\varphi_{2}$ and

$$
\begin{aligned}
& w=\{u, v\}, \quad x=\frac{1}{2}\{u, w\}, \quad y=\frac{1}{3}\{u, x\}, \quad z=\{v, y\} . \\
& 3 \alpha+2 \beta: z
\end{aligned}
$$


The Poisson bracket and the action of $s_{1}, s_{2}$ are determined by the following tables.

\begin{tabular}{c|cccccc}
$\{\}$, & $u$ & $v$ & $w$ & $x$ & $y$ & $z$ \\
\hline$u$ & 0 & $w$ & $2 x$ & $3 y$ & 0 & 0 \\
$v$ & $-w$ & 0 & 0 & 0 & $z$ & 0 \\
$w$ & $-2 x$ & 0 & 0 & $-3 z$ & 0 & 0 \\
$x$ & $-3 y$ & 0 & $3 z$ & 0 & 0 & 0 \\
$y$ & 0 & $-z$ & 0 & 0 & 0 & 0 \\
$z$ & 0 & 0 & 0 & 0 & 0 & 0
\end{tabular}

\begin{tabular}{c||cc} 
& $a$ & $b$ \\
\hline$s_{1}$ & $-a$ & $a+b$ \\
$s_{2}$ & $a+3 b$ & $-b$
\end{tabular}

\begin{tabular}{c||cccccc} 
& $u$ & $v$ & $w$ & $x$ & $y$ & $z$ \\
\hline$s_{1}$ & $u$ & $v+\frac{a w}{u}+\frac{a^{2} x}{u^{2}}+\frac{a^{3} y}{u^{3}}$ & $w+\frac{2 a x}{u}+\frac{3 a^{2} y}{u^{2}}$ & $x+\frac{3 a y}{u}$ & $y$ & $z$ \\
$s_{2}$ & $u-\frac{b w}{v}$ & $v$ & $w$ & $x$ & $y+\frac{b z}{v}$ & $z$
\end{tabular}

The nilpotent Poisson algebra $\mathcal{A}_{0}=\mathbb{C}[u, v, w, x, y, z]$ defines a group of canonical transformations, isomorphic to the Weyl group $W\left(G_{2}\right)$, on the field of rational functions $\mathcal{K}=\mathbb{C}(a, b, u, v, w, x, y, z)$.

The $\tau$-cocycle in this case is given as follows.

$$
\begin{aligned}
& \phi_{1}\left(\Lambda_{1}\right)=\phi_{s_{2}}\left(\Lambda_{1}\right)=1, \quad \phi_{s_{1}}\left(\Lambda_{1}\right)=\phi_{s_{1} s_{2}}\left(\Lambda_{1}\right)=u \\
& \phi_{s_{2} s_{1}}\left(\Lambda_{1}\right)=\phi_{s_{2} s_{1} s_{2}}\left(\Lambda_{1}\right)=u v-b w, \\
& \phi_{s_{1} s_{2} s_{1}}\left(\Lambda_{1}\right)=\phi_{s_{1} s_{2} s_{1} s_{2}}\left(\Lambda_{1}\right)=u^{3} v-b u^{2} w-a(a+2 b) u x-a^{2}(2 a+3 b) y \\
& \phi_{s_{2} s_{1} s_{2} s_{1}}\left(\Lambda_{1}\right)=\phi_{s_{2} s_{1} s_{2} s_{1} s_{2}}\left(\Lambda_{1}\right) \\
& =u^{3} v^{2}-2 b u^{2} v w-(a+b)(a+3 b) u v x+b^{2} u w^{2} \\
& \quad-(a+3 b)^{2}(2 a+3 b) v y+b(a+b)(a+3 b) w x-b(a+3 b)^{2}(2 a+3 b) z \\
& \phi_{s_{1} s_{2} s_{1} s_{2} s_{1}}\left(\Lambda_{1}\right)=\phi_{s_{1} s_{2} s_{1} s_{2} s_{1} s_{2}}\left(\Lambda_{1}\right) \\
& =u^{4} v^{2}-2 b u^{3} v w-\left(2 a^{2}+6 a b+3 b^{2}\right) u^{2} v x+b^{2} u^{2} w^{2}-(2 a+3 b)^{3} u v y \\
& \quad+b\left(2 a^{2}+6 a b+3 b^{2}\right) u w x-(a+b)(a+3 b)(2 a+3 b)^{2} u z \\
& \quad-a(a+2 b)(2 a+3 b)^{2} w y+a(a+b)(a+2 b)(a+3 b) x^{2} \\
& \phi_{1}\left(\Lambda_{2}\right)=\phi_{s_{1}}\left(\Lambda_{2}\right)=1, \quad \phi_{s_{2}}\left(\Lambda_{2}\right)=\phi_{s_{2} s_{1}}\left(\Lambda_{2}\right)=v \\
& \phi_{s_{1} s_{2}}\left(\Lambda_{2}\right)=\phi_{s_{1} s_{2} s_{1}}\left(\Lambda_{2}\right)=u^{3} v+a u^{2} w+a^{2} u x+a^{3} y \\
& \phi_{s_{2} s_{1} s_{2}}\left(\Lambda_{2}\right)=\phi_{s_{2} s_{1} s_{2} s_{1}}\left(\Lambda_{2}\right) \\
& =u^{3} v^{3}+a u^{2} v^{2} w+(a+3 b)^{2} u v^{2} x-b(2 a+3 b) u v w^{2} \\
& \quad+(a+3 b)^{3} v^{2} y-b(a+3 b)^{2} v w x+b(a+3 b)^{3} v z+b^{2}(a+2 b) w^{3}
\end{aligned}
$$




$$
\begin{aligned}
& \phi_{s_{1} s_{2} s_{1} s_{2}}\left(\Lambda_{2}\right)=\phi_{s_{1} s_{2} s_{1} s_{2} s_{1}}\left(\Lambda_{2}\right) \\
& =u^{6} v^{3}+2 a u^{5} v^{2} w+\left(5 a^{2}+12 a+9 b^{2}\right) u^{4} v^{2} x-b(4 a+3 b) u^{4} v w^{2} \\
& +(2 a+3 b)^{2}(5 a+3 b) u^{3} v^{2} y-b\left(8 a^{2}+15 a b+9 b^{2}\right) u^{3} v w x \\
& +(a+b)(2 a+3 b)^{3} u^{3} v z+2 b^{2}(a+b) u^{3} w^{3}+a(2 a+3 b)^{2}(5 a+3 b) u^{2} v w y \\
& -a\left(5 a^{3}+24 a^{2} b+36 a b^{2}+18 b^{3}\right) u^{2} v x^{2}+3 a b^{2}(a+b) u^{2} w^{2} x \\
& +a(a+b)(2 a+3 b)^{3} u^{2} w z-a^{2}(a+3 b)(2 a+3 b)^{2} u v x y \\
& +2 a^{2}(a+b)(2 a+3 b)^{2} u w^{2} y-a^{2}(a+b)\left(2 a^{2}+6 a b+3 b^{2}\right) u w x^{2} \\
& +a^{2}(a+b)(2 a+3 b)^{2} x z-a^{3}(2 a+3 b)^{3} v y^{2}+a^{3}(a+b)(2 a+3 b)^{2} w x y \\
& -a^{3}(a+b)^{2}(a+2 b) x^{3}+a^{3}(a+b)(2 a+3 b)^{3} y z \\
& \phi_{s_{2} s_{1} s_{2} s_{1} s_{2}}\left(\Lambda_{2}\right)=\phi_{s_{2} s_{1} s_{2} s_{1} s_{2} s_{1}}\left(\Lambda_{2}\right) \\
& =u^{6} v^{4}+2 a u^{5} v^{3} w+\left(5 a^{2}+18 a b+18 b^{2}\right) u^{4} v^{3} x-6 b(a+b) u^{4} v^{2} w^{2} \\
& +(2 a+3 b)^{2}(5 a+12 b) u^{3} v^{3} y-3 b\left(4 a^{2}+13 a b+12 b^{2}\right) u^{3} v^{2} w x \\
& +2(a+3 b)^{2}(2 a+3 b)^{2} u^{3} v^{2} z+2 b^{2}(3 a+4 b) u^{3} v w^{3} \\
& +a(2 a+3 b)^{2}(5 a+12 b) u^{2} v^{2} w y-(a+3 b)\left(5 a^{3}+21 a^{2} b+27 a b^{2}+9 b^{3}\right) u^{2} v^{2} x^{2} \\
& +3 b^{2}\left(3 a^{2}+8 a b+6 b^{2}\right) u^{2} v w^{2} x+2 a(a+3 b)^{2}(2 a+3 b)^{2} u^{2} v w z \\
& -b^{3}(2 a+3 b) u^{2} w^{4}-a(a+3 b)^{2}(2 a+3 b)^{2} u v^{2} x y \\
& +a(2 a+3 b)^{2}\left(2 a^{2}+6 a b+3 b^{2}\right) u v w^{2} y \\
& -a(a+3 b)\left(2 a^{2}+6 a^{2} b+3 a b^{2}-3 b^{3}\right) u v w x^{2} \\
& +2(a+3 b)^{2}(2 a+3 b)^{2}\left(a^{2}+3 a b+3 b^{2}\right) u v x z-a b^{3}(2 a+3 b) u w^{3} x \\
& -2 b(a+b)(a+3 b)^{2}(2 a+3 b)^{2} u w^{2} z-(a+3 b)^{3}(2 a+3 b)^{3} v^{2} y^{2} \\
& +(a+3 b)^{2}(2 a+3 b)^{2}\left(a^{2}+6 a b+6 b^{2}\right) v w x y-(a+b)(a+2 b)^{2}(a+3 b)^{3} v x^{3} \\
& +a(a+3 b)^{3}(2 a+3 b)^{3} v y z-b(a+2 b)^{2}(2 a+3 b)^{3} w^{3} y \\
& +b(a+b)(a+3 b)(2 a+3 b)\left(a^{2}+3 a b+3 b^{2}\right) w^{2} x^{2} \\
& -a b(a+b)(a+3 b)^{2}(2 a+3 b)^{2} w x z+b(a+b)(a+3 b)^{3}(2 a+3 b)^{3} z^{2}
\end{aligned}
$$

We remark that

$$
\begin{aligned}
& \phi_{s_{1} s_{2} s_{1} s_{2} s_{1}}\left(\Lambda_{1}\right)=\varphi_{1} s_{1} \varphi_{2} s_{2} \varphi_{1}^{2} s_{1} \varphi_{2} s_{2}\left(\varphi_{1}\right), \quad \text { and } \\
& \phi_{s_{2} s_{1} s_{2} s_{1} s_{2}}\left(\Lambda_{2}\right)=\varphi_{2} s_{2} \varphi_{1}^{3} s_{1} \varphi_{2}^{2} s_{2} \varphi_{1}^{3} s_{1}\left(\varphi_{2}\right)
\end{aligned}
$$

are invariant with respect to $s_{2}$ and $s_{1}$, respectively. 


\section{Realization of Weyl groups}

In this section, we give a proof of Theorems 1.1 and 1.2. In fact we prove these two theorems as a consequence of their formal version (Theorem 3.2 below).

\subsection{Formal version}

Fixing a GCM $A=\left(a_{i j}\right)_{i, j \in I}$, let $\mathcal{R}$ be a Poisson which contains a set of invertible elements $\left(\varphi_{i}\right)_{i \in I}$ such that

$$
\operatorname{ad}_{\{\}}\left(\varphi_{i}\right)^{-a_{i j}+1}\left(\varphi_{j}\right)=0 \quad(i \neq j) .
$$

We denote by $\mathcal{R}[[\lambda]]$ the ring of formal power series in the $\lambda$-variables $\left(\lambda_{i}\right)_{i \in I}$ with coefficients in $\mathcal{R}$. The Poisson bracket on $\mathcal{R}$ extends naturally to $\mathcal{R}[[\lambda]]$ by the trivial action on the $\lambda$-variables. We also use the same notation $r_{i}: \mathcal{R}[[\lambda]] \rightarrow$ $\mathcal{R}[[\lambda]]$ for the formal completion of the $\mathcal{R}$-linear automorphism $r_{i}: \mathcal{R}[\lambda] \rightarrow \mathcal{R}[\lambda]$ such that $r_{i}\left(\lambda_{j}\right)=\lambda_{j}-a_{j i} \lambda_{i}(j \in I)$, For each $i \in I$, we define the linear mapping $s_{i}: \mathcal{R}[[\lambda]] \rightarrow \mathcal{R}[[\lambda]]$ by

$$
s_{i}=\exp \left(\frac{\lambda_{i}}{\varphi_{i}} \operatorname{ad}_{\{\}}\left(\varphi_{i}\right)\right) \circ r_{i} \quad(i \in I) .
$$

In the following we set

$$
X_{i}=\frac{1}{\varphi_{i}} \operatorname{ad}_{\{\}}\left(\varphi_{i}\right) \quad(i \in I),
$$

so that $R_{i}(t)=\exp \left(t X_{i}\right)$ and $s_{i}=R_{i}\left(\lambda_{i}\right) \circ r_{i}$.

Lemma 3.1 For each $i \in I, s_{i}$ defines a canonical transformation of $\mathcal{R}[[\lambda]]$ :

$$
s_{i}(\varphi \psi)=s_{i}(\varphi) s_{i}(\psi), \quad s_{i}(\{\varphi, \psi\})=\left\{s_{i}(\varphi), s_{i}(\psi)\right\},
$$

for any $\varphi, \psi \in \mathcal{R}[[\lambda]]$.

This lemma is a consequence of the fact that the derivations $X_{i}$ have the property

$$
X_{i}(\{f, g\})=\left\{X_{i}(f), g\right\}+\left\{f, X_{i}(g)\right\} \quad(f, g \in \mathcal{R}) .
$$

We denote by $\mathcal{W}=\left\langle s_{i}(i \in I)\right\rangle$ the free group generated by the symbols $s_{i}$ $(i \in I)$, and by $\rho: \mathcal{W} \rightarrow W$ the homomorphism defined by $\rho\left(s_{i}\right)=r_{i}(i \in I)$. At this stage, we have a group homomorphism $\mathcal{W} \rightarrow \operatorname{Aut}(\mathcal{R}[[\lambda]])$, and this action, restricted to the $\lambda$-variables, is factored through $\rho: \mathcal{W} \rightarrow W$.

We also consider the ring of Laurent polynomials $\mathcal{R}[[\lambda]]\left[\tau^{ \pm 1}\right]$ in the $\tau$ variables, and extend the action of $s_{i}$ to $\mathcal{R}[[\lambda]]\left[\tau^{ \pm 1}\right]$ by

$$
s_{i}\left(\tau_{j}\right)=\tau_{j} \quad(i \neq j), \quad s_{i}\left(\tau_{i}\right)=\varphi_{i} \tau_{i} \prod_{k \in I} \tau_{k}^{-a_{k i}} .
$$

In this setting, we have a group homomorphism $\mathcal{W} \rightarrow \operatorname{Aut}\left(\mathcal{R}[[\lambda]]\left[\tau^{ \pm 1}\right]\right)$. Our goal is to prove 
Theorem 3.2 The automorphisms $s_{i}(i \in I)$ of $\mathcal{R}[[\lambda]]\left[\tau^{ \pm 1}\right]$, defined as above, satisfy the fundamental relations for the Weyl group $W(A)$ :

$$
s_{i}^{2}=1, \quad\left(s_{i} s_{j}\right)^{m_{i j}}=1 \quad(i \neq j) .
$$

We show how Theorem 3.2 implies Theorems 1.1 and 1.2. With the notation of Section 1, we take the localization $\mathcal{R}=\mathcal{A}_{0}\left[\varphi_{j}^{-1}(j \in I)\right]$. In order to establish Theorem 1.1, we have only to check the validity of equalities $s_{i}^{2}(\psi)=1$ and $\left(s_{i} s_{j}\right)^{m_{i j}}(\psi)=1$ for any $\psi \in \mathcal{A}_{0}$. By the definition of $s_{j}(j \in I)$, the elements $s_{i}^{2}(\psi),\left(s_{i} s_{j}\right)^{m_{i j}}(\psi)$ of $\mathcal{K}=Q\left(\mathcal{A}_{0}\right)(\lambda)=Q(\mathcal{R})(\lambda)$ are regular at $\lambda=0$. Hence the equalities $s_{i}^{2}(\psi)=1$ and $\left(s_{i} s_{j}\right)^{m_{i j}}(\psi)=1$ follow from those in the ring of formal power series $\mathcal{R}[[\lambda]]$ which are guaranteed by Theorem 3.2. Also, Theorem 1.2. concerning the action of $s_{i}$ on $\mathcal{K}\left[\tau^{ \pm 1}\right]$, follows similarly from the equalities $s_{i}^{2}\left(\tau_{j}\right)=\tau_{j}$ and $\left(s_{i} s_{j}\right)^{m_{i j}}\left(\tau_{j}\right)=\tau_{j}$ in $\mathcal{R}[[\lambda]]\left[\tau^{ \pm 1}\right]$.

\section{$3.2 \quad \tau$-Cocycle and braid relations on $\tau$-functions}

We first check the equality $s_{i}^{2}=1$ on $\mathcal{R}[[\lambda]]$ for each $i \in I$. In fact, with the $R$-operator notation $R_{i}(t)=\exp \left(t X_{i}\right)$, one has

$$
s_{i}^{2}=R_{i}\left(\lambda_{i}\right) r_{i} R_{i}\left(\lambda_{i}\right) r_{i}=R_{i}\left(\lambda_{i}\right) R_{i}\left(-\lambda_{i}\right) r_{i}^{2}=1,
$$

since $R_{i}(-t)=\exp \left(-t X_{i}\right)=R_{i}(t)^{-1}$. It is also easy to see $s_{i}^{2}\left(\tau_{j}\right)=\tau_{j}$ for all $j \in I$.

Instead of the Coxeter relations $\left(s_{i} s_{j}\right)^{m_{i j}}=1$, we will verify the following braid relations:

$$
\begin{aligned}
& \text { (0) }\left(a_{i j}, a_{j i}\right)=(0,0) \quad \Longrightarrow \quad s_{i} s_{j}=s_{j} s_{i} \text {, } \\
& \text { (1) }\left(a_{i j}, a_{j i}\right)=(-1,-1) \quad \Longrightarrow \quad s_{i} s_{j} s_{i}=s_{j} s_{i} s_{j} \text {, } \\
& \text { (2) }\left(a_{i j}, a_{j i}\right)=(-2,-1) \quad \Longrightarrow \quad s_{i} s_{j} s_{i} s_{j}=s_{j} s_{i} s_{j} s_{i} \text {, } \\
& \text { (3) }\left(a_{i j}, a_{j i}\right)=(-3,-1) \Longrightarrow s_{i} s_{j} s_{i} s_{j} s_{i} s_{j}=s_{j} s_{i} s_{j} s_{i} s_{j} s_{i} \text {, }
\end{aligned}
$$

which corresponds to the root systems of rank 2 of type $2 A_{1}, A_{2}, B_{2}$ and $G_{2}$, respectively. Note that the validity of each of these braid relations on $\mathcal{R}[[\lambda]]$ is equivalent to the corresponding Yang-Baxter equation in (1.13) for $R_{j}(t)$. Since the commutativity $s_{i} s_{j}=s_{j} s_{i}$ for the case $a_{i j}=a_{j i}=0$ is immediate, we will mainly consider the other three cases.

Lemma 3.3 The following identities hold in $\mathcal{R}[[\lambda]]$.

(1) If $\left(a_{i j}, a_{j i}\right)=(-1,-1)$,

$$
\varphi_{j} s_{j}\left(\varphi_{i}\right)=s_{i} \varphi_{j} s_{j}\left(\varphi_{i}\right), \quad \varphi_{i} s_{i}\left(\varphi_{j}\right)=s_{j} \varphi_{i} s_{i}\left(\varphi_{j}\right) .
$$

(2) If $\left(a_{i j}, a_{j i}\right)=(-2,-1)$,

$$
\varphi_{i} s_{i} \varphi_{j} s_{j}\left(\varphi_{i}\right)=s_{j} \varphi_{i} s_{i} \varphi_{j} s_{j}\left(\varphi_{i}\right), \quad \varphi_{j} s_{j} \varphi_{i}^{2} s_{i}\left(\varphi_{j}\right)=s_{i} \varphi_{j} s_{j} \varphi_{i}^{2} s_{i}\left(\varphi_{j}\right) .
$$

(3) If $\left(a_{i j}, a_{j i}\right)=(-3,-1)$,

$$
\begin{aligned}
& \varphi_{i} s_{i} \varphi_{j} s_{j} \varphi_{i}^{2} s_{i} \varphi_{j} s_{j}\left(\varphi_{i}\right)=s_{j} \varphi_{i} s_{i} \varphi_{j} s_{j} \varphi_{i}^{2} s_{i} \varphi_{j} s_{j}\left(\varphi_{i}\right), \\
& \varphi_{j} s_{j} \varphi_{i}^{3} s_{i} \varphi_{j}^{2} s_{j} \varphi_{i}^{3} s_{i}\left(\varphi_{j}\right)=s_{i} \varphi_{j} s_{j} \varphi_{i}^{3} s_{i} \varphi_{j}^{2} s_{j} \varphi_{i}^{3} s_{i}\left(\varphi_{j}\right) .
\end{aligned}
$$


In this lemma, we regard $\varphi_{i}$ and $\varphi_{j}$ as multiplication operators. The two identities in the case of $\left(a_{i j}, a_{j i}\right)=(-1,-1)$, for example, should be read as

$$
\varphi_{j} s_{j}\left(\varphi_{i}\right)=s_{i}\left(\varphi_{j}\right) s_{i} s_{j}\left(\varphi_{i}\right), \quad \varphi_{i} s_{i}\left(\varphi_{j}\right)=s_{j}\left(\varphi_{i}\right) s_{j} s_{i}\left(\varphi_{j}\right) .
$$

We will not write down the corresponding formulas for the other two cases. Note that, in each case, the exponents of $\varphi_{i}, \varphi_{j}$ in the two formulas correspond to the coefficients of $a$ and $b$ in the Yang-Baxter equation (1.13), respectively. The six equalities in Lemma 3.3 can be verified by direct computation, by using the automorphisms $s_{1}, s_{2}$ as in the examples of the previous section. (In fact, we made use of a computer algebra system to check Lemma 3.3 for $B_{2}$ and $G_{2}$.) We will explain now how Lemma 3.3 imply the Yang-Baxter equations (1.13) and the braid relations (3.50).

In order to clarify the meaning of Lemma 3.3 , we use the terminology of $\tau$ cocycle. With the notation of formal exponentials $\tau^{\Lambda}(\Lambda \in L)$, for each $w \in \mathcal{W}$, one can define the elements $\phi_{w}(\Lambda) \in \mathcal{R}[[\lambda]](\Lambda \in L)$ by the formula

$$
w\left(\tau^{\Lambda}\right)=\phi_{w}(\Lambda) \tau^{\rho(w) . \Lambda} \quad(w \in \mathcal{W}, \Lambda \in L) .
$$

By the definition, these $\phi_{w}(\Lambda)$ have the following cocycle property.

Proposition 3.4 (1) The elements $\phi_{w}(\Lambda)$ are determined uniquely by

$$
\begin{aligned}
& \phi_{1}(\Lambda)=1, \quad \phi_{s_{i}}(\Lambda)=\phi_{s_{i}^{-1}}(\Lambda)=\varphi_{i}^{\left\langle h_{i}, \Lambda\right\rangle} \quad(i \in I), \\
& \phi_{w}\left(\Lambda+\Lambda^{\prime}\right)=\phi_{w}(\Lambda) \phi_{w}\left(\Lambda^{\prime}\right), \quad \phi_{w w^{\prime}}(\Lambda)=\phi_{w}\left(\rho\left(w^{\prime}\right) . \Lambda\right) w\left(\phi_{w^{\prime}}(\Lambda)\right),
\end{aligned}
$$

for any $w, w^{\prime} \in \mathcal{W}$ and $\Lambda, \Lambda^{\prime} \in L$.

(2) For any $w=s_{j_{1}} s_{j_{2}} \cdots s_{j_{p}} \in \mathcal{W}$ and $\Lambda \in L, \phi_{w}(\Lambda)$ is expressed as

$$
\begin{aligned}
& \phi_{w}(\Lambda)=\prod_{k=1}^{p} s_{j_{1}} \cdots s_{j_{k-1}}\left(\varphi_{j_{k}}^{\left\langle h_{j_{k}}, r_{j_{k+1}} \cdots r_{j_{p}} \Lambda\right\rangle}\right) \\
& =\varphi_{j_{1}}^{\left\langle h_{j_{1}}, r_{j_{2}} \cdots r_{j_{p}} \Lambda\right\rangle} s_{j_{1}} \varphi_{j_{2}}^{\left\langle h_{j_{2}}, r_{j_{3}} \cdots r_{j_{p}} \Lambda\right\rangle} \cdots \\
& \quad \cdot s_{j_{1}} \cdots s_{j_{p-2}} \varphi_{j_{p-1}}^{\left\langle h_{j_{p-1}}, r_{j_{p}} \Lambda\right\rangle} s_{j_{1}} \cdots s_{j_{p-1}}\left(\varphi_{j_{p}}^{\left\langle h_{j_{p}}, \Lambda\right\rangle}\right) .
\end{aligned}
$$

At this stage, we only know that $\phi$ is a 1-cocycle of $\mathcal{W}$ with coefficients in the $\mathcal{W}$-bimodule $\operatorname{Hom}\left(L, \mathcal{K}^{\times}\right)$.

The formulas of Lemma 3.3 are understood as relations among $\phi_{w}(\Lambda)$ for certain $w \in \mathcal{W}$ and $\Lambda \in L$. For example, let us take two indices $i, j \in I$ such that $\left(a_{i j}, a_{j i}\right)=(-1,-1)$. Then, as a special case of formula (3.57), we have

$$
\begin{aligned}
& \phi_{s_{j} s_{i} s_{j}}\left(\Lambda_{i}\right)=\varphi_{j} s_{j}\left(\varphi_{i}\right), \quad \phi_{s_{i} s_{j} s_{i}}\left(\Lambda_{i}\right)=s_{i} \varphi_{j} s_{j}\left(\varphi_{i}\right), \\
& \phi_{s_{i} s_{j} s_{i}}\left(\Lambda_{j}\right)=\varphi_{i} s_{i}\left(\varphi_{j}\right), \quad \phi_{s_{j} s_{i} s_{j}}\left(\Lambda_{j}\right)=s_{j} \varphi_{i} s_{i}\left(\varphi_{j}\right) .
\end{aligned}
$$

Note also that $\phi_{s_{i} s_{j} s_{i}}\left(\Lambda_{k}\right)=1$ for $k \neq i, j$. Hence, Lemma 3.3 (1) implies

$$
\phi_{s_{i} s_{j} s_{i}}\left(\Lambda_{k}\right)=\phi_{s_{j} s_{i} s_{j}}\left(\Lambda_{k}\right) \quad(k \in I) .
$$

In the same way, Lemma 3.3 can be reformulated as follows. 
Lemma 3.5 For each $i, j \in I$, the following identities hold in $\mathcal{R}[[\lambda]]$.

(1) If $\left(a_{i j}, a_{j i}\right)=(-1,-1), \quad \phi_{s_{i} s_{j} s_{i}}(\Lambda)=\phi_{s_{j} s_{i} s_{j}}(\Lambda) \quad(\Lambda \in L)$.

(2) If $\left(a_{i j}, a_{j i}\right)=(-2,-1), \quad \phi_{s_{i} s_{j} s_{i} s_{j}}(\Lambda)=\phi_{s_{j} s_{i} s_{j} s_{i}}(\Lambda) \quad(\Lambda \in L)$.

(3) If $\left(a_{i j}, a_{j i}\right)=(-3,-1), \quad \phi_{s_{i} s_{j} s_{i} s_{j} s_{i} s_{j}}(\Lambda)=\phi_{s_{j} s_{i} s_{j} s_{i} s_{j} s_{i}}(\Lambda) \quad(\Lambda \in L)$.

Note that Lemma 3.5 implies that the braid relations are valid on the $\tau$ functions.

Corollary 3.6 When $a_{i j} a_{j i}=0,1,2$, or 3 , one has

$$
s_{i} s_{j} s_{i} \cdots\left(\tau^{\Lambda}\right)=s_{j} s_{i} s_{j} \cdots\left(\tau^{\Lambda}\right) \quad\left(m_{i j} \text { factors of } s_{k} \text { 's on each side }\right)
$$

for all $\Lambda \in L$.

\subsection{Braid relations on $\mathcal{R}[[\lambda]]$}

We now proceed to the braid relations on the Poisson algebra $\mathcal{R}[[\lambda]]$. For the argument below, it is convenient to extend the Poisson algebra $\mathcal{R}$ to

$$
\widetilde{\mathcal{R}}=\mathbb{C}\left[\log \varphi_{j}(j \in I)\right] \otimes_{\mathbb{C}} \mathcal{R},
$$

by adjoining the formal logarithms of the elements $\varphi_{i}(i \in I)$. The Poisson bracket $\{$,$\} extends naturally to \widetilde{\mathcal{R}}$ and $\widetilde{\mathcal{R}}[[\lambda]]$ by

$$
\begin{gathered}
\left\{\left(\log \varphi_{j}\right)^{m}, \psi\right\}=m\left(\log \varphi_{j}\right)^{m-1} \frac{1}{\varphi_{j}}\left\{\varphi_{j}, \psi\right\} \\
\left\{\left(\log \varphi_{i}\right)^{m},\left(\log \varphi_{j}\right)^{n}\right\}=m n\left(\log \varphi_{i}\right)^{m-1}\left(\log \varphi_{j}\right)^{n-1} \frac{1}{\varphi_{i} \varphi_{j}}\left\{\varphi_{i}, \varphi_{j}\right\}
\end{gathered}
$$

for any $i, j \in I, \psi \in \widetilde{\mathcal{R}}$ and $m, n=0,1,2, \ldots$. Consequently, one can define the action of $s_{i}$ on $\widetilde{\mathcal{R}}[[\lambda]]$ by

$$
s_{i}=\exp \left(\lambda_{i} X_{i}\right) \circ r_{i}, \quad X_{i}=\operatorname{ad}_{\{\}}\left(\log \varphi_{i}\right)=\frac{1}{\varphi_{i}} \operatorname{ad}_{\{\}}\left(\varphi_{i}\right) \quad(i \in I) .
$$

Note that each Yang-Baxter equation in (1.13) takes the form

$$
\begin{aligned}
& \exp \left(\mu_{1} X_{i}\right) \exp \left(\mu_{2} X_{j}\right) \cdots \exp \left(\mu_{m} X_{\ell}\right) \\
& \quad=\exp \left(\mu_{m} X_{j}\right) \exp \left(\mu_{m-1} X_{i}\right) \cdots \exp \left(\mu_{1} X_{k}\right)
\end{aligned}
$$

where $k=i$ or $j$, and $\ell=j$ or $i$, according to the parity of $m=3,4,6 ; \mu_{1}, \ldots, \mu_{m}$ are linear functions in $a=\lambda_{i}, b=\lambda_{j}$ for $k=1, \ldots, m$. Denote the left hand side and the right hand side of (3.64), regarded as functions of $\lambda=\left(\lambda_{i}\right)_{i \in I}$, by $\Phi(\lambda)$ and by $\Psi(\lambda)$, respectively. Since $\Phi(0)=\Psi(0)$ in each case, equality (3.64) reduces to proving

$$
\partial_{\lambda_{i}}(\Phi(\lambda)) \Phi(\lambda)^{-1}=\partial_{\lambda_{i}}(\Psi(\lambda)) \Psi(\lambda)^{-1} \quad(i \in I) .
$$


For an arbitrary element $\Lambda \in L$, we define the derivation with respect to $\Lambda$ by

$$
\partial_{\Lambda}=\sum_{i \in I}\left\langle h_{i}, \Lambda\right\rangle \partial_{\lambda_{i}}
$$

We will derive the formula

$$
\partial_{\Lambda}(\Phi(\lambda)) \Phi(\lambda)^{-1}=\partial_{\Lambda}(\Psi(\lambda)) \Psi(\lambda)^{-1} \quad(\Lambda \in L)
$$

from a general statement for operators defined as certain product of canonical transformations of the form $R_{i}(t)=\exp \left(t X_{i}\right)$.

For the moment, we fix an arbitrary element $\Lambda \in L$. For each operator $P=P(\lambda) \in \operatorname{End}_{\mathbb{C}}(\widetilde{\mathcal{R}})[[\lambda]]$ with invertible leading term $P(0)$, we set

$$
L_{P}=\partial_{\Lambda}(P(\lambda)) P(\lambda)^{-1} .
$$

Then, as to the composition of two operators, we have

$$
L_{P Q}=L_{P}+P(\lambda) L_{Q} P(\lambda)^{-1} .
$$

If $P$ is expressed as

$$
P(\lambda)=\exp (a(\lambda) X), \quad X=\operatorname{ad}_{\{\}}(f)
$$

with some $f \in \widetilde{\mathcal{R}}$ and a linear function $a=a(\lambda)$, then we have

$$
\begin{aligned}
& L_{P}=\partial_{\Lambda}(a) X=\operatorname{ad}_{\{\}}\left(\partial_{\Lambda}(a) f\right), \\
& P(\lambda) L_{Q} P(\lambda)^{-1}=\exp (a X) L_{Q} \exp (-a X)=\exp (a \operatorname{ad}(X))\left(L_{Q}\right) .
\end{aligned}
$$

Suppose furthermore that $L_{Q}=\operatorname{ad}_{\{\}}(g(\lambda))$ for some $g(\lambda) \in \widetilde{\mathcal{R}}[[\lambda]]$. Since $X=\operatorname{ad}_{\{\}}(f)$, by the Jacobi identity for the Poisson bracket, we have

$$
\exp (a \operatorname{ad}(X))\left(L_{Q}\right)=\exp (a \operatorname{ad}(X))\left(\operatorname{ad}_{\{\}}(g(\lambda))\right)=\operatorname{ad}_{\{\}}(\exp (a X) g(\lambda)) .
$$

Hence we obtain the following expression for $L_{P Q}$ :

$$
L_{P Q}=\operatorname{ad}_{\{\}}\left(\partial_{\Lambda}(a) f+\exp (a X) g(\lambda)\right) .
$$

We make use of these observations to analyze the action of $\mathcal{W}$.

For each $w=s_{j_{1}} s_{j_{2}} \cdots s_{j_{p}} \in \mathcal{W}$, we define the operator $G_{w}(\lambda)$ by

$$
w=G_{w}(\lambda) r, \quad G_{w}(\lambda)=\exp \left(\mu_{1} X_{j_{1}}\right) \exp \left(\mu_{2} X_{j_{2}}\right) \cdots \exp \left(\mu_{p} X_{j_{p}}\right),
$$

where $r=\rho(w)=r_{j_{1}} \cdots r_{j_{p}}$ and $\mu_{k}=r_{j_{1}} \cdots r_{j_{k-1}}\left(\lambda_{j_{k}}\right)$ for $k=1, \ldots, p$. In the following we use the multiplicative notation

$$
\log \left(\varphi_{i}^{a} \varphi_{j}^{b} \cdots\right)=a \log \varphi_{i}+b \log \varphi_{j}+\cdots \quad(a, b, \cdots \in \mathbb{Z}) .
$$


Also, for a power series in the form

$$
f(\lambda)=f_{0}+\sum_{|\nu|>0} f_{\nu} \lambda^{\nu} \quad\left(f_{\nu} \in \mathcal{R}\right)
$$

(with the multi-index notation) such that $f_{0}$ is a product of $\varphi_{j}$ 's, we write

$$
\log f(\lambda)=\log f_{0}+\log \left(1+f_{0}^{-1} \sum_{|\nu|>0} f_{\nu} \lambda^{\nu}\right),
$$

understanding the last term by its Taylor expansion.

Proposition 3.7 For any element $w=s_{j_{1}} \cdots s_{j_{p}} \in \mathcal{W}$ and $\Lambda \in L$, one has

$$
\partial_{\Lambda}\left(G_{w}(\lambda)\right) G_{w}(\lambda)^{-1}=-\operatorname{ad}_{\{\}}\left(\log \phi_{w}\left(\rho(w)^{-1} \cdot \Lambda\right)\right) .
$$

Proof. If $P=\exp \left(\lambda_{j} X_{j}\right)$,

$$
L_{P}=\operatorname{ad}_{\{\}}\left(\left\langle h_{j}, \Lambda\right\rangle \log \varphi_{j}\right)=\operatorname{ad}_{\{\}}\left(\log \varphi_{j}^{\left\langle h_{j}, \Lambda\right\rangle}\right)=-\operatorname{ad}_{\{\}}\left(\log \varphi_{j}^{\left\langle h_{j}, r_{j} \Lambda\right\rangle}\right) .
$$

This proves the case where $p=1$. When $w=s_{j_{1}} \cdots s_{j_{p}}$ and $r=\rho(w)=$ $r_{j_{1}} \cdots r_{j_{p}}$, by using the expression (3.74), we set

$$
P=\exp \left(\lambda_{j_{1}} X_{j_{1}}\right), \quad Q=\exp \left(\mu_{2} X_{j_{2}}\right) \cdots \exp \left(\mu_{p} X_{j_{p}}\right)
$$

so that $G_{w}=P Q$. By the induction hypothesis, we have

$$
\partial_{\Lambda}\left(G_{w^{\prime}}\right) G_{w^{\prime}}^{-1}=-\operatorname{ad}_{\{\}}\left(\log \phi_{w^{\prime}}\left(r^{\prime-1} . \Lambda\right)\right)
$$

for $w^{\prime}=s_{j_{1}} w=s_{j_{2}} \cdots s_{j_{p}}, r^{\prime}=r_{j_{1}} r=r_{j_{2}} \cdots r_{j_{p}}$. . Since $Q=r_{j_{1}} G_{w^{\prime}} r_{j_{1}}$, we compute

$$
\partial_{\Lambda}(Q) Q^{-1}=r_{j_{1}} \partial_{r_{j_{1}} \Lambda}\left(G_{w^{\prime}}\right) G_{w^{\prime}}^{-1} r_{j_{1}}=-\operatorname{ad}_{\{\}}\left(\log r_{j_{1}}\left(\phi_{w^{\prime}}\left(r^{-1} \Lambda\right)\right)\right) .
$$

Hence by (3.73) we obtain

$$
\begin{aligned}
& \partial_{\Lambda}\left(G_{w}\right) G_{w}{ }^{-1}=L_{P Q} \\
& =-\operatorname{ad}_{\{\}}\left(\log \varphi_{j_{1}}^{\left\langle h_{j_{1}}, r_{j_{1}} \Lambda\right\rangle}+\log \exp \left(\lambda_{j_{1}} X_{j_{1}}\right) r_{j_{1}}\left(\phi_{w^{\prime}}\left(r^{-1} \Lambda\right)\right)\right) \\
& =-\operatorname{ad}_{\{\}}\left(\log \left(\varphi_{j_{1}}^{\left\langle h_{j_{1}}, r^{\prime} r^{-1} \Lambda\right\rangle} s_{j_{1}}\left(\phi_{w^{\prime}}\left(r^{-1} \Lambda\right)\right)\right)\right) \\
& =-\operatorname{ad}_{\{\}}\left(\log \phi_{w}\left(r^{-1} . \Lambda\right)\right)
\end{aligned}
$$

for any $\Lambda \in L$.

Q.E.D.

When $\left(a_{i j}, a_{j i}\right)=(-k,-1)$ for $k=1,2,3$, we set

$$
\Phi(\lambda)=G_{s_{i} s_{j} s_{i} \ldots}(\lambda), \quad \Psi(\lambda)=G_{s_{j} s_{i} s_{j} \ldots}(\lambda) .
$$


Then Proposition 3.7 implies

$$
\begin{aligned}
& \partial_{\Lambda}(\Phi(\lambda)) \Phi(\lambda)^{-1}=-\operatorname{ad}_{\{\}}\left(\log \phi_{s_{i} s_{j} s_{i} \ldots}\left(r^{-1} . \Lambda\right)\right) \\
& \partial_{\Lambda}(\Psi(\lambda)) \Psi(\lambda)^{-1}=-\operatorname{ad}_{\{\}}\left(\log \phi_{s_{j} s_{i} s_{j} \ldots} \ldots\left(r^{-1} . \Lambda\right)\right),
\end{aligned}
$$

where $r=r_{i} r_{j} r_{i} \cdots=r_{j} r_{i} r_{j} \ldots$. Since $\phi_{s_{i} s_{j} s_{i} \ldots}=\phi_{s_{j} s_{i} s_{j} \ldots}$ by Lemma 3.5, we see

$$
\partial_{\Lambda}(\Phi(\lambda)) \Phi(\lambda)^{-1}=\partial_{\Lambda}(\Psi(\lambda)) \Psi(\lambda)^{-1}
$$

for any $\Lambda \in L$. Since $\Phi(0)=\Psi(0)$, we have $\Phi(\lambda)=\Psi(\lambda)$, namely

$$
G_{s_{i} s_{j} s_{i} \ldots}(\lambda)=G_{s_{j} s_{i} s_{j} \ldots}(\lambda)
$$

which proves the Yang-Baxter equation of (1.13). Since $s_{i} s_{j} s_{i} \ldots=G_{s_{i} s_{j} s_{i} \ldots r}$ and $s_{j} s_{i} s_{j} \ldots=G_{s_{j} s_{i} s_{j} \ldots r}$, we obtain the braid relation of $(3.50)$ on $\mathcal{R}[[\lambda]]$ as desired. Since we have already seen the validity of braid relation on $\tau$-functions in Corollary 3.6, this completes the proof of Theorems 3.2.

Remark: In the formulation of Section 1, we assumed for simplicity that $\mathcal{A}_{0}$ is generated as a Poisson algebra, by a set of nonzero elements $\varphi_{i}(i \in I)$ satisfying the Serre relations. As can be seen from the proof of this section, for the validity of Theorems 1.1 and 1.2, we have only to assume that the following three conditions are satisfied:

(i) $\mathcal{A}_{0}$ has no zerodivisors.

(ii) $\mathcal{A}_{0}$ contains a set of nonzero elements $\varphi_{i}(i \in I)$ such that $\operatorname{ad}_{\{\}}\left(\varphi_{i}\right)^{-a_{i j}+1} \varphi_{j}=0(i \neq j)$.

(iii) The action of $\operatorname{ad}_{\{\}}\left(\varphi_{i}\right)$ on $\mathcal{A}_{0}$ is locally nilpotent for each $i \in I$.

\section{Lie theoretic background}

In this section, we assume that the GCM $A=\left(a_{i j}\right)_{i j \in I}$ is symmetrizable and that the indexing set $I$ is finite. We fix a set of nonzero rational numbers $\epsilon_{i}$ $(i \in I)$ such that $a_{i j} \epsilon_{j}=a_{j i} \epsilon_{i}$ for $i, j \in I$. We will explain below a geometric background of our realization of the Weyl group $W=W(A)$ in the language of Kac-Moody Lie algebras and Kac-Moody groups.

\subsection{Birational action of $W$ on the Borel subalgebra}

As to Kac-Moody Lie algebras, we will basically follow the notation of Kac [5]. Let $\mathfrak{g}=\mathfrak{g}(A)$ be the Kac-Moody Lie algebra associated with the symmetrizable generalized Cartan matrix $A$, and consider the triangular decomposition $\mathfrak{g}=$ $\mathfrak{n} \oplus \mathfrak{h} \oplus \mathfrak{n}_{-}$, where $\mathfrak{n}$ (resp. $\mathfrak{n}_{-}$) is the nilpotent Lie subalgebras of $\mathfrak{g}$ generated by $e_{i}\left(\right.$ resp. $f_{i}$ ). We denote by $\mathfrak{g}=\bigoplus_{n \in \mathbb{Z}} \mathfrak{g}_{n}$ the gradation of $\mathfrak{g}$ with respect to the degree (height) such that $\operatorname{deg} e_{i}=1, \operatorname{deg} f_{i}=-1(i \in I)$ and $\operatorname{deg} h=0$ $(h \in \mathfrak{h})$. Note that each $\mathfrak{g}_{n}$ is finite dimensional and that $\mathfrak{n}=\oplus_{n>0} \mathfrak{g}_{n}, \mathfrak{h}=\mathfrak{g}_{0}$, $\mathfrak{n}_{-}=\oplus_{n<0} \mathfrak{g}_{n}$. We denote by $\mathfrak{g}=\mathfrak{h} \oplus \sum_{\alpha \in \Delta} \mathfrak{g}_{\alpha}$ the root space decomposition of 
$\mathfrak{g}$ with respect to the Cartan subalgebra $\mathfrak{h}$, so that $\mathfrak{g}_{n}=\sum_{\mathrm{ht}(\alpha)=n} \mathfrak{g}_{\alpha}(n \in \mathbb{Z}$, $n \neq 0)$.

The symmetrizable Kac-Moody Lie algebra $\mathfrak{g}=\mathfrak{g}(A)$ has an ad-invariant symmetric bilinear form $(\mid): \mathfrak{g} \times \mathfrak{g} \rightarrow \mathbb{C}$ which induces nondegenerate pairings $\mathfrak{h} \times \mathfrak{h} \rightarrow \mathbb{C}$ and $\mathfrak{g}_{-\alpha} \times \mathfrak{g}_{\alpha} \rightarrow \mathbb{C}(\alpha \in \Delta)$. We take the normalization of $(\mid)$ such that

$$
\left(h_{i} \mid h_{j}\right)=a_{i j} \epsilon_{j}, \quad\left(e_{i} \mid f_{j}\right)=\delta_{i j} \epsilon_{j} \quad(i, j \in I) .
$$

Through this symmetric bilinear form, we have an isomorphism $\iota: \mathfrak{g} \stackrel{\sim}{\rightarrow} \mathfrak{g}^{*}$ of $\mathbb{C}$-vector spaces such that $\iota(y)(x)=(y \mid x)$ for $y, x \in \mathfrak{g}$, where $\mathfrak{g}^{*}=\oplus_{n \in \mathbb{Z}} \mathfrak{g}_{n}^{*}$ is the graded dual of $\mathfrak{g}$. We define the bracket $\{\}:, \mathfrak{g}^{*} \times \mathfrak{g}^{*} \rightarrow \mathfrak{g}^{*}$ by

$$
\left\{\iota(y), \iota\left(y^{\prime}\right)\right\}=-\iota\left(\left[y, y^{\prime}\right]\right) \quad\left(y, y^{\prime} \in \mathfrak{g}\right) .
$$

This bracket naturally extends to a Poisson bracket of $\operatorname{Sym}\left(\mathfrak{g}^{*}\right)$. Note that each element of the Poisson algebra $\operatorname{Sym}\left(\mathfrak{g}^{*}\right)$ is regarded naturally as a polynomial function on $\mathfrak{g}$. If $\varphi=\iota(y)$ and $\psi=\iota\left(y^{\prime}\right)\left(y, y^{\prime} \in \mathfrak{g}\right)$, the ad-invariance of $(\mid)$ implies

$$
\left(\operatorname{ad}_{\{\}}(\varphi) \psi\right)(x)=-\left(\operatorname{ad}(y) y^{\prime} \mid x\right)=\left(y^{\prime} \mid \operatorname{ad}(y) x\right)=\psi(\operatorname{ad}(y) x)
$$

for any $x \in \mathfrak{g}$. Hence we have

Lemma 4.1 Let $y \in \mathfrak{g}$ and set $\varphi=\iota(y) \in \mathfrak{g}^{*}$. Then, for any $\psi \in \operatorname{Sym}\left(\mathfrak{g}^{*}\right)$, one has

$$
\left(\exp \left(t \operatorname{ad}_{\{\}}(\varphi)\right) \psi\right)(x)=\psi(\exp (t \operatorname{ad}(y)) x) \quad(x \in \mathfrak{g}),
$$

where $t$ is a formal parameter.

One has only to verify (4.89) for $\psi \in \mathfrak{g}^{*}$, since the both sides of (4.89) is multiplicative with respect to $\psi$. This equality for $\psi=\iota\left(y^{\prime}\right)\left(y^{\prime} \in \mathfrak{g}\right)$ follows from (4.88).

We consider now the formal completion $\widehat{\mathfrak{n}}=\lim _{k} \mathfrak{n} / \operatorname{ad}(\mathfrak{n})^{k} \mathfrak{n}$ of the nilpotent subalgebra $\mathfrak{n} \subset \mathfrak{g}$ with respect to the height, and set $\widehat{\mathfrak{b}}=\mathfrak{h} \oplus \widehat{\mathfrak{n}}$. The coordinate rings of $\mathfrak{h}, \widehat{\mathfrak{n}}$ and $\widehat{\mathfrak{b}}$ are defined by

$$
\mathcal{O}(\mathfrak{h})=\operatorname{Sym}\left(\mathfrak{h}^{*}\right), \quad \mathcal{O}(\widehat{\mathfrak{n}})=\operatorname{Sym}\left(\mathfrak{n}^{*}\right) \quad \text { and } \quad \mathcal{O}(\widehat{\mathfrak{b}})=\operatorname{Sym}\left(\mathfrak{h}^{*} \oplus \mathfrak{n}^{*}\right),
$$

respectively, where $\mathfrak{n}^{*}=\oplus_{n=1}^{\infty} \mathfrak{g}_{n}^{*}$ stands for the graded dual of $\mathfrak{n}$. Note that $\iota: \mathfrak{g} \stackrel{\sim}{\rightarrow} \mathfrak{g}^{*}$ induces the isomorphism $\mathfrak{n}_{-} \stackrel{\sim}{\rightarrow} \mathfrak{n}^{*}$ and that $\mathcal{O}(\widehat{\mathfrak{n}})=\operatorname{Sym}\left(\mathfrak{n}^{*}\right)$ can be regarded as a Poisson subalgebra of $\operatorname{Sym}\left(\mathfrak{g}^{*}\right)$. Let $\left\{\varphi_{i}\right\}_{i \in I}$ be the basis of $\mathfrak{g}_{1}^{*}$ dual to the basis $\left\{e_{i}\right\}_{i \in I}$ of $\mathfrak{g}_{1}: \varphi_{i}\left(e_{j}\right)=\delta_{i j}(i, j \in I)$. Then $\mathcal{O}(\widehat{\mathfrak{n}})$ is generated as a Poisson algebra by $\left\{\varphi_{i}\right\}_{i \in I}$, and these generators satisfy the Serre relations

$$
\operatorname{ad}_{\{\}}\left(\varphi_{i}\right)^{-a_{i j}+1}\left(\varphi_{j}\right)=0 \quad(i \neq j)
$$

with respect to the Poisson bracket, since $\varphi_{i}=\iota\left(f_{i}\right) / \epsilon_{i}(i \in I)$. Note also that the simple roots $\alpha_{i}(i \in I)$ are regarded as functions on $\widehat{\mathfrak{b}}$ of height 0 . Hence the coordinate ring $\mathcal{O}(\widehat{\mathfrak{b}})$ contains the subalgebra $\mathbb{C}[\alpha] \otimes \mathcal{O}(\widehat{\mathfrak{n}})$. 
In this setting we explain a procedure to define a birational action of the Weyl group $W(A)$ on the Borel subalgebra $\widehat{\mathfrak{b}}$. For each $i$, consider the adjoint action of the 1-parameter subgroup $\exp \left(t f_{i}\right)(t \in \mathbb{C})$ on $\widehat{\mathfrak{b}}$. Note that $\exp \left(t \operatorname{ad}\left(f_{i}\right)\right)$ is well defined as a mapping $\widehat{\mathfrak{b}} \rightarrow \mathbb{C} f_{i} \oplus \widehat{\mathfrak{b}}$, since, for each $\beta \in \Delta_{+}$, there are only a finite number of positive roots of the form $\beta+n \alpha_{i}(n=0,1,2, \ldots)$. For an element $x \in \widehat{\mathfrak{b}}$ given, we try to find a value of $t$ such that

$$
\operatorname{Ad}\left(\exp \left(t f_{i}\right)\right) x=\exp \left(t \operatorname{ad}\left(f_{i}\right)\right) x \in \widehat{\mathfrak{b}} .
$$

A general element $x \in \widehat{\mathfrak{b}}$ can be expressed in the form

$$
x=h+\sum_{j \in I} c_{j} e_{j}+(\text { components of height } \geq 2),
$$

where $h \in \mathfrak{h}$ and $c_{j}=\varphi_{j}(x)(j \in I)$. By a simple computation, we see that $\exp \left(t \operatorname{ad}\left(f_{i}\right)\right) x$ takes the form

$$
t\left(\left\langle h, \alpha_{i}\right\rangle-t c_{i}\right) f_{i}+\left(h-t c_{i} h_{i}\right)+(\text { components of height } \geq 1) .
$$

Hence we have a nontrivial solution

$$
t=\frac{\left\langle h, \alpha_{i}\right\rangle}{c_{i}}=\frac{\alpha_{i}(x)}{\varphi_{i}(x)},
$$

provided that $c_{i}=\varphi_{i}(x) \neq 0$. Note that, when $t=\left\langle h, \alpha_{i}\right\rangle / c_{i}$, one has

$$
\exp \left(t \operatorname{ad}\left(f_{i}\right)\right) x=h-\left\langle h, \alpha_{i}\right\rangle h_{i}+(\text { components of height } \geq 1) .
$$

From this argument, for each $i \in I$, we obtain a regular mapping

$$
\sigma_{i}: x \mapsto \operatorname{Ad}\left(\exp \left(t_{i}(x) f_{i}\right)\right) x: \widehat{\mathfrak{b}} \backslash\left\{\varphi_{i}=0\right\} \rightarrow \widehat{\mathfrak{b}},
$$

where $t_{i}=\alpha_{i} / \varphi_{i}$. Passing to the coordinate ring, we denote by $\sigma_{i}^{*}$ the automorphism of the field $\mathcal{K}(\widehat{\mathfrak{b}})$ of rational functions on $\widehat{\mathfrak{b}}$, corresponding to the birational mapping $\sigma_{i}: \widehat{\mathfrak{b}} \cdots \rightarrow \widehat{\mathfrak{b}}$.

Proposition 4.2 For each $i \in I$, the automorphism $\sigma_{i}^{*}$ of $\mathcal{K}(\widehat{\mathfrak{b}})$ is characterized by the following two properties:

$$
\begin{aligned}
\sigma_{i}^{*}(\lambda) & =\lambda-\alpha_{i}\left\langle h_{i}, \lambda\right\rangle & & \left(\lambda \in \mathfrak{h}^{*}\right), \\
\sigma_{i}^{*}(\psi) & =\exp \left(\frac{\lambda_{i}}{\varphi_{i}} \operatorname{ad}_{\{\}}\left(\varphi_{i}\right)\right) \psi & & (\psi \in \mathcal{O}(\widehat{\mathfrak{n}})),
\end{aligned}
$$

where $\lambda_{i}=\epsilon_{i} \alpha_{i}$.

The first equality for $\lambda \in \mathfrak{h}^{*}$ is a consequence of the expression (4.96). If $\psi \in \mathcal{O}(\widehat{\mathfrak{n}})$, by Lemma 4.1 one has

$$
\begin{aligned}
\sigma_{i}^{*}(\psi)(x) & =\psi\left(\exp \left(t_{i}(x) \operatorname{ad}\left(f_{i}\right)\right) x\right) \\
& =\left(\exp \left(\epsilon_{i} t_{i}(x) \operatorname{ad}_{\{\}}\left(\varphi_{i}\right)\right) \psi\right)(x) \\
& =\left(\exp \left(\epsilon_{i} t_{i} \operatorname{ad}_{\{\}}\left(\varphi_{i}\right)\right) \psi\right)(x)
\end{aligned}
$$


for any $x \in \widehat{\mathfrak{b}} \backslash\left\{\varphi_{i}=0\right\}$. This proves the second statement, since $\epsilon_{i} t_{i}=\epsilon_{i} \alpha_{i} / \varphi_{i}$.

These automorphisms $\sigma_{i}^{*}(i \in I)$ are essentially the same as $s_{i}(i \in I)$ in Theorem 1.1 for the nilpotent Poisson algebra $\mathcal{A}_{0}=\mathcal{O}(\widehat{\mathfrak{n}})$. In fact, the polynomial ring $\mathbb{C}[\lambda]$ with $\lambda_{i}=\epsilon_{i} \alpha_{i}$ is a subalgebra of $\mathcal{O}\left(\mathfrak{h}^{*}\right)$, and each automorphism $\sigma_{i}^{*}$ coincides with $s_{i}$ on the subfield $\mathcal{K}=Q(\mathbb{C}[\lambda] \otimes \mathcal{O}(\widehat{\mathfrak{n}}))$ of $\mathcal{K}(\widehat{\mathfrak{b}})$.

Although the construction of $\sigma_{i}^{*}$ gives an origin of our birational automorphisms $s_{i}$, it does not explain why these automorphisms satisfy the fundamental relations for the generators of the Weyl group $W=W(A)$. In the following subsections, we will show that the birational automorphism $\sigma_{i}$ of $\widehat{\mathfrak{b}}$ arise naturally from the birational dressing action of a lift of the Weyl group on the Borel subgroup, induced through the Gauss decomposition in the Kac-Moody group. This construction also provides an alternative proof of Theorem 1.1 for the case when the GCM $A$ is symmetrizable and $\mathcal{A}_{0}=\mathcal{O}(\widehat{\mathfrak{n}})$.

\subsection{Birational action of $\dot{W}$ on the Borel subgroup}

We denote by $\mathfrak{g}^{\prime}=\mathfrak{n} \oplus \mathfrak{h}^{\prime} \oplus \mathfrak{n}$ - be the subalgebra of $\mathfrak{g}$, generated by the Chevalley generators $e_{i}, h_{i}, f_{i}(i \in I)$, where $\mathfrak{h}^{\prime}=\bigoplus_{i \in I} \mathbb{C} h_{i}$. In the following we will make use of a variant of Kac-Moody group $G$ for the Lie algebra $\mathfrak{g}^{\prime}$. Although there are several variants of Kac-Moody groups (到, [5], [7], [10]), we will work now with the one as in Slodowy 10]. (In the argument below, we can also use the infinite dimensional scheme of Kashiwara [5], since we do not need the group structure on the whole $G$.)

We first recall some properties of $G$ which will be used in our argument.

(1) The group $G$ contains the Borel subgroup

$$
B=T \ltimes U, \quad T=\operatorname{Spec}(\mathbb{C}[L]), \quad U=\exp (\widehat{\mathfrak{n}})
$$

where $L=\operatorname{Hom}_{\mathbb{Z}}\left(Q^{\vee}, \mathbb{Z}\right)$, and $\widehat{\mathfrak{n}}=\varliminf_{k} \mathfrak{n} / \operatorname{ad}(\mathfrak{n})^{k}(\mathfrak{n})$ is the completion of $\mathfrak{n}$ with respect to the height.

(2) For each $i \in I, G$ contains a parabolic subgroup $P_{i} \supset B$ associated with the simple root $\alpha_{i}$. It has the Levi decomposition

$$
P_{i}=G_{i} \ltimes U_{i}, \quad U_{i}=\exp \left(\widehat{\mathfrak{n}}_{i}\right)
$$

where $G_{i}$ the group of the Lie algebra $\mathfrak{g}_{i}^{\prime}=\mathbb{C} e_{i} \oplus \mathfrak{h}^{\prime} \oplus \mathbb{C} f_{i}$ with the Cartan subgroup $T$, and $\widehat{\mathfrak{n}}_{i}=\lim _{k} \mathfrak{n}_{i} / \operatorname{ad}(\mathfrak{n})^{k}\left(\mathfrak{n}_{i}\right)$ is the completion of $\mathfrak{n}_{i}=\bigoplus_{\alpha \in \Delta_{+} \backslash\left\{\alpha_{i}\right\}} \mathfrak{g}_{\alpha}$ with respect to the height.

(3) The group $G$ has a subgroup $U_{-}$such that $U_{-} \cap B=\{1\}$ and that all the 1-parameter subgroups associated with the negative real roots are contained in $U_{-}$.

(4) For each dominant integral weight $\Lambda \in P_{+}$, (i.e., $\Lambda \in \mathfrak{h}^{*}$ such that $\left\langle h_{i}, \Lambda\right\rangle \in$ $\mathbb{Z}_{\geq 0}$ for all $\left.i \in I\right)$, the irreducible $\mathfrak{g}$-module $L(\Lambda)$ with highest weight $\Lambda$ can be integrated to a $G$-module.

For each $i \in I$, we set

$$
\dot{s}_{i}=\exp \left(-e_{i}\right) \exp \left(f_{i}\right) \exp \left(-e_{i}\right) \in G_{i} \subset P_{i}
$$


and denote by $\dot{W}$ the subgroup of $G$ generated by $\dot{s}_{i}(i \in I)$. These elements satisfy $\dot{s}_{j}^{4}=1$ and the braid relations

$$
\dot{s}_{i} \dot{s}_{j} \dot{s}_{i} \cdots=\dot{s}_{j} \dot{s}_{i} \dot{s}_{j} \cdots \quad\left(m_{i j} \text { factors on each side }\right)
$$

in $G$, for any $i, j \in I$ with $a_{i j} a_{j i}=0,1,2,3$. In what follows, we denote by $W=W(A)$ the Weyl group on the generators $s_{i}(i \in I)$. We have a natural group homomorphism $\dot{W} \rightarrow W$ which maps $\dot{s}_{i}$ to $s_{i}(i \in I)$.

We now express a general element of the Borel subgroup $B=T \ltimes U$ as

$$
b=\tau \exp (\xi), \quad \tau=\prod_{i \in I} \tau_{i}^{h_{i}} \in T, \quad \xi=\sum_{n=1}^{\infty} \xi_{n} \in \widehat{\mathfrak{n}} \quad\left(\xi_{n} \in \mathfrak{g}_{n}\right) .
$$

In this decomposition, the coordinate $\operatorname{ring} \mathcal{O}(U)$ of $U$ is defined as the symmetric algebra $\operatorname{Sym}\left(\mathfrak{n}^{*}\right)$ of the graded dual of $\mathfrak{n}$. For a general element $\xi \in \widehat{\mathfrak{n}}$, we express the component $\xi_{1}$ of height 1 as $\xi_{1}=\sum_{i \in I} \psi_{i} e_{i}$, regarding $\psi_{i}(i \in I)$ as the part of coordinates of $U$ in height 1 . These $\psi_{i}(i \in I)$ form a $\mathbb{C}$-basis of $\mathfrak{g}_{1}^{*}$ dual to the $\mathbb{C}$-basis $e_{i}(i \in I)$ of $\mathfrak{g}_{1}$. Similarly we regard $\tau_{i}(i \in I)$ as the coordinates of the Cartan subgroup $T \simeq\left(\mathbb{C}^{\times}\right)^{I}$, and use the notation $\tau^{\Lambda}=\prod_{i \in I} \tau_{i}^{\left\langle h_{i}, \Lambda\right\rangle} \in \mathcal{O}(T)$ for any $\Lambda \in L$. Note that the coordinate $\operatorname{ring} \mathcal{O}(B)$ of $B$ is represented as the ring of Laurent polynomials $\mathcal{O}(U)\left[\tau^{ \pm 1}\right]$ in $\tau_{i}(i \in I)$ with coefficients in $\mathcal{O}(U)=\operatorname{Sym}\left(\mathfrak{n}^{*}\right)$.

Let $\dot{w}$ be an arbitrary element of $\dot{W}$. Suppose that $\exp (\xi) \in U$ is generic so that $\exp (\xi) \dot{w} \in U_{-} B$. Then, for $b=\tau \exp (\xi) \in B$ with an arbitrary $\tau \in T$, the product $b \dot{w}$ can be decomposed uniquely in the form

$$
b \dot{w}=g^{-1} \widetilde{b}, \quad g \in U_{-}, \quad \widetilde{b} \in B .
$$

This type of Gauss decomposition (Birkhoff decomposition) is determined by carrying out the Gauss decomposition for the right action of $\dot{s}_{i}{ }^{ \pm 1}(i \in I)$ repeatedly. For the argument below, we examine the case of $\dot{w}=\dot{s}_{i}$ in some detail.

Proposition 4.3 For each $i \in I$, any element $b=\tau \exp (\xi)$ with $\psi_{i} \neq 0$ is decomposed uniquely in the form $b \dot{s}_{i}=g_{i}^{-1} \widetilde{b}$ with $g_{i} \in U_{-}$and $\widetilde{b}=\widetilde{\tau} \exp (\widetilde{\xi}) \in B$. The element $g$ and $\widetilde{\tau}$ are given by

$$
g_{i}=\exp \left(\frac{-1}{\psi_{i} \tau^{\alpha_{i}}} f_{i}\right), \quad \widetilde{\tau}=\tau \psi_{i}^{h_{i}},
$$

where $\tau^{\alpha_{i}}=\prod_{k \in I} \tau_{k}^{\left\langle h_{k}, \alpha_{i}\right\rangle}=\prod_{k \in I} \tau_{k}^{a_{k i}}$. Furthermore, the correspondence $\xi \rightarrow \widetilde{\xi}$ is represented by an algebra homomorphism $\mathcal{O}(U) \rightarrow \mathcal{O}(U)\left[\psi_{i}^{-1}\right]$.

The Gauss decomposition of $b \dot{s}_{i}$ is determined by employing the Levi decomposition $P_{i}=G_{i} \ltimes U_{i}$ and the following lemma in $G_{i}$. 
Lemma 4.4 Let $a, b \in \mathbb{C}$ and $c \in \mathbb{C}^{\times}$. For each $i \in I$, one has the identity

$$
\exp \left(a e_{i}\right) c^{h_{i}} \exp \left(b f_{i}\right)=\exp \left(y f_{i}\right) z^{h_{i}} \exp \left(x e_{i}\right)
$$

in $G_{j}$ unless $a b+c^{2}=0$, where

$$
x=\frac{a}{a b+c^{2}}, \quad z=\frac{a b+c^{2}}{c}, \quad y=\frac{b}{a b+c^{2}} .
$$

Proof of Proposition 4.3. By decomposing $b=\tau \exp (\xi) \in B$ in the form

$$
b=\tau \exp \left(\psi_{i} e_{i}\right) \exp \left(\xi^{\prime}\right), \quad \xi^{\prime} \in \widehat{\mathfrak{n}}_{i},
$$

we get

$$
b \dot{s}_{i}=\tau \exp \left(\psi_{i} e_{i}\right) \exp \left(\xi^{\prime}\right) \dot{s}_{i}=\tau \exp \left(\psi_{i} e_{i}\right) \dot{s}_{i} \exp \left(\xi^{\prime \prime}\right),
$$

where $\xi^{\prime \prime}=\operatorname{Ad}\left(\dot{s}_{i}^{-1}\right)\left(\xi^{\prime}\right)$. Note that $\exp \left(-\operatorname{ad}\left(f_{i}\right)\right): \widehat{\mathfrak{n}}_{i} \rightarrow \widehat{\mathfrak{n}}_{i}$ is well defined since, for any $\beta \in \Delta_{+} \backslash\left\{\alpha_{i}\right\}$, there are only a finite number of positive roots in the form $\beta+n \alpha_{i}(n \geq 0)$. Then by Lemma 4.4 we have

$$
\begin{aligned}
\exp \left(\psi_{i} e_{i}\right) \dot{s}_{i} & =\exp \left(\psi_{i} e_{i}\right) \exp \left(-e_{i}\right) \exp \left(f_{i}\right) \exp \left(-e_{i}\right) \\
& =\exp \left(\left(\psi_{i}-1\right) e_{i}\right) \exp \left(f_{i}\right) \exp \left(-e_{i}\right) \\
& =\exp \left(\frac{1}{\psi_{i}} f_{i}\right) \psi_{i}^{h_{i}} \exp \left(\left(1-\frac{1}{\psi_{i}}\right) e_{i}\right) \exp \left(-e_{i}\right) \\
& =\exp \left(\frac{1}{\psi_{i}} f_{i}\right) \psi_{i}^{h_{i}} \exp \left(\frac{-1}{\psi_{i}} e_{i}\right) .
\end{aligned}
$$

Hence,

$$
\begin{aligned}
b \dot{s}_{i} & =\tau \exp \left(\frac{1}{\psi_{i}} f_{i}\right) \psi_{i}^{h_{i}} \exp \left(\frac{-1}{\psi_{i}} e_{i}\right) \exp \left(\xi^{\prime \prime}\right) \\
& =\exp \left(\frac{1}{\psi_{i} \tau^{\alpha_{i}}} f_{i}\right) \tau \psi_{i}^{h_{i}} \exp (\widetilde{\xi}),
\end{aligned}
$$

where $\exp (\widetilde{\xi})=\exp \left(\frac{-1}{\psi_{i}} e_{i}\right) \exp \left(\xi^{\prime \prime}\right)$. This computation implies that $\widetilde{\xi}$ is regular on $\psi_{i} \neq 0$, and $g=\exp \left(\frac{-1}{\psi_{i} \tau^{\alpha_{i}}} f_{i}\right), \widetilde{\tau}=\tau \psi_{i}^{h_{i}}$, as desired.

Q.E.D.

We now consider the Gauss decomposition of $b \dot{w}$ for an arbitrary $\dot{w} \in \dot{W}$ :

$$
b \dot{w}=g^{-1} \widetilde{b}, \quad g \in U_{-}, \quad \widetilde{b} \in B,
$$

for generic $b \in B$. We denote the two elements $g$ and $\widetilde{b}$ above by $g(\dot{w}, b) \in U_{-}$ and $b * \dot{w} \in B$, respectively. From this Gauss decomposition of $b \dot{w}$, we obtain a mapping $* \dot{w}$ from an open dense subset of $B$, which sends generic $b \in B$ to $b * \dot{w}=g(\dot{w}, b) b \dot{w} \in B$. Note that the uniqueness of Gauss decomposition implies the following 1-cocycle relation for $g(\dot{w}, b)$ :

$$
g(1, b)=1, \quad g\left(\dot{w}_{1} \dot{w}_{2}, b\right)=g\left(\dot{w}_{2}, b * \dot{w}_{1}\right) g\left(w_{1}, b\right)
$$

for any $\dot{w} \in \dot{W}$ and for generic $b \in B$. From Proposition 1.3, we see that, for each $i \in I, g_{i}=g\left(\dot{s}_{i}, b\right)$ is defined for $b=\tau \exp (\xi)$ with $\psi_{i} \neq 0$ by

$$
g_{i}=g\left(\dot{s}_{i}, b\right)=\exp \left(\frac{-1}{\psi_{i} \tau^{\alpha_{j}}} f_{i}\right) .
$$


The element $g(\dot{w}, b)$ for general $\dot{w} \in \dot{W}$ is determined inductively from $g\left(\dot{s}_{j}, b\right)$ by the cocycle relation (4.114). Also, we obtain a birational right action of $\dot{W}$ on $B$ :

$$
b * 1=b, \quad b *\left(\dot{w}_{1} \dot{w}_{2}\right)=\left(b * \dot{w}_{1}\right) * \dot{w}_{2}
$$

for any $\dot{w}_{1}, \dot{w}_{2} \in \dot{W}$ and for generic $b \in B$. In terms of the coordinate rings, this birational mapping $* \dot{w}: B \cdots \rightarrow B$ defines an algebra automorphism

$$
R_{\dot{w}}: \mathcal{K}(U)\left[\tau^{ \pm}\right] \rightarrow \mathcal{K}(U)\left[\tau^{ \pm}\right],
$$

$\mathcal{K}(U)$ being the field of rational functions on $U$, such that

$$
\left(R_{\dot{w}} \psi\right)(b)=\psi(b * \dot{w})=\psi(g(\dot{w}, b) b \dot{w})
$$

for any $\psi \in \mathcal{K}(U)\left[\tau^{ \pm 1}\right]$ and for generic $b \in B$. Note also that

$$
R_{1}=1, \quad R_{\dot{w}_{1} \dot{w}_{2}}=R_{\dot{w}_{1}} R_{\dot{w}_{2}} \quad\left(\dot{w}_{1}, \dot{w}_{2} \in \dot{W}\right) .
$$

These $R_{\dot{w}}$ give a realization of $\dot{W}$ as a group of automorphisms of the ring $\mathcal{K}(U)\left[\tau^{ \pm 1}\right]$ of Laurent polynomials. We remark that, if $\dot{w}=\dot{s}_{j_{1}} \cdots \dot{s}_{j_{p}}$, the homomorphism $R_{\dot{w}}$ is determined as the composition $R_{\dot{w}}=R_{\dot{s}_{j_{1}}} \cdots R_{\dot{s}_{j_{p}}}$.

\subsection{Passage to the Borel subalgebra}

We now consider to transfer the birational action of $\dot{W}$ on the Borel subgroup $B$ to that on the Borel subalgebra $\widehat{\mathfrak{b}}$ by means of the adjoint action.

We say that an element $h$ of the Cartan subalgebra $\mathfrak{h}$ is regular if $\langle h, \alpha\rangle \neq 0$ for any positive root $\alpha \in \Delta_{+}$. We denote the set of all regular elements in $\mathfrak{h}$ by $\mathfrak{h}_{\text {reg }}$, and set $\widehat{\mathfrak{b}}_{\text {rs }}=\mathfrak{h}_{\text {reg }}+\widehat{\mathfrak{n}}$, so that $\mathcal{O}\left(\widehat{\mathfrak{b}}_{\text {rs }}\right)=\mathcal{O}\left(\mathfrak{h}_{\text {reg }}\right) \otimes \mathcal{O}(\widehat{\mathfrak{n}}), \mathcal{O}\left(\mathfrak{h}_{\text {reg }}\right)=$ $\operatorname{Sym}\left(\mathfrak{h}^{*}\right)\left[\alpha^{-1}\left(\alpha \in \Delta_{+}\right)\right]$.

Proposition 4.5 The mapping

$$
\mu: B \times \mathfrak{h}_{\text {reg }} \rightarrow T \times \widehat{\mathfrak{b}}_{\mathrm{rs}}:(b=\tau \exp (\xi), h) \mapsto(\tau, \operatorname{Ad}(b)(h))
$$

gives an isomorphism of affine spaces.

Proof. Under the condition that $h \in \mathfrak{h}$ is regular, for $\tau \in T$ and $x \in \widehat{\mathfrak{n}}$ given, one can find a unique $\xi \in \widehat{\mathfrak{n}}$ such that $\operatorname{Ad}(\tau \exp (\xi)) h=h+x$, i.e.,

$$
\exp (\operatorname{ad}(\xi))(h)=h+\operatorname{Ad}\left(\tau^{-1}\right)(x)
$$

inductively with respect to the height. In fact, by the root space decomposition

$$
\xi=\sum_{\alpha \in \Delta_{+}} \xi_{\alpha}, \quad x=\sum_{\alpha \in \Delta_{+}} x_{\alpha} \quad\left(\xi_{\alpha}, x_{\alpha} \in \mathfrak{g}_{\alpha}\right),
$$


the equation above is equivalently rewritten into the recurrence formulas

$$
\langle h, \alpha\rangle \xi_{\alpha}+\sum_{k=2}^{h t(\alpha)} \frac{1}{k !} \sum_{\beta_{1}+\cdots+\beta_{k}=\alpha}\left\langle h, \beta_{k}\right\rangle \operatorname{ad}\left(\xi_{\beta_{1}}\right) \cdots \operatorname{ad}\left(\xi_{\beta_{k-1}}\right)\left(\xi_{\beta_{k}}\right)=-\tau^{-\alpha} x_{\alpha}
$$

for $\alpha \in \Delta_{+}$. In terms of the coordinate rings, these recurrence formulas imply that the mapping $(b, h) \rightarrow \operatorname{Ad}(b)(h)$ is represented the $\mathcal{O}(\mathfrak{h})$-algebra homomorphism $\mathcal{O}(\widehat{\mathfrak{b}})=\mathcal{O}(\widehat{\mathfrak{n}}) \otimes \mathcal{O}(\mathfrak{h}) \rightarrow \mathcal{O}(B) \otimes \mathcal{O}(\mathfrak{h})$ and that it induces an isomorphism

$$
\begin{aligned}
\mu^{*}: & \mathcal{O}(T) \otimes \mathcal{O}\left(\widehat{\mathfrak{b}}_{\mathrm{rs}}\right)=\mathcal{O}(T) \otimes \mathcal{O}\left(\mathfrak{h}_{\mathrm{reg}}\right) \otimes \mathcal{O}(\widehat{\mathfrak{n}}) \\
& \stackrel{\sim}{\rightarrow} \mathcal{O}(B) \otimes \mathcal{O}\left(\mathfrak{h}_{\mathrm{reg}}\right)=\mathcal{O}(T) \otimes \mathcal{O}\left(\mathfrak{h}_{\mathrm{reg}}\right) \otimes \mathcal{O}(U)
\end{aligned}
$$

of $\mathcal{O}(T) \otimes \mathcal{O}\left(\mathfrak{h}_{\text {reg }}\right)$-algebras. This proves Proposition.

Q.E.D.

The recurrence formulas (4.123) imply the inclusions

$$
\begin{aligned}
\mu^{*}(\mathcal{O}(\widehat{\mathfrak{n}})) & \subset \mathcal{O}(\mathfrak{h}) \otimes \mathcal{O}(U) \otimes \mathcal{O}(T), \\
\left(\mu^{*}\right)^{-1}(\mathcal{O}(U)) & \subset \mathcal{O}\left(\mathfrak{h}_{\mathrm{reg}}\right) \otimes \mathcal{O}(\widehat{\mathfrak{n}}) \otimes \mathcal{O}(T) .
\end{aligned}
$$

We remark that, as a special case of (4.123) when $\alpha=\alpha_{i}$, we have

$$
\left\langle h, \alpha_{i}\right\rangle \psi_{i}=-\tau^{-\alpha_{i}} \varphi_{i} \quad(h \in \mathfrak{h}, i \in I) .
$$

It means that, under the identification of Proposition 4.5, we have

$$
\psi_{i}=-\frac{\varphi_{i}}{\alpha_{i}} \tau^{-\alpha_{i}}, \quad \varphi_{i}=-\alpha_{i} \psi_{i} \tau^{\alpha_{i}}, \quad \text { where } \tau^{\alpha_{i}}=\prod_{k \in I} \tau_{k}^{a_{k i}} .
$$

(In the expression $\tau^{\alpha_{i}}, \alpha_{i}$ is treated as a simple root. Otherwise, $\alpha_{i}$ is regarded as a function on $\mathfrak{h}$.)

We now consider to transfer the birational action of $\dot{W}$ from $B$ to $\widehat{\mathfrak{b}}_{\mathrm{rs}}$ by the isomorphism of Proposition 4.5. The product space $B \times \mathfrak{h}_{\text {reg }}$ admits the right birational action of $\dot{W}$ defined by

$$
(b, h) * \dot{w}=\left(b * \dot{w}, \operatorname{Ad}(\dot{w})^{-1} h\right)=\left(g(\dot{w}, b) b \dot{w}, \operatorname{Ad}(\dot{w})^{-1} h\right)
$$

for generic $b \in B$ and for $h \in \mathfrak{h}_{\text {reg. }}$. We denote by the same symbol $* \dot{w}$ the birational action of $\dot{W}$ on $T \times \widehat{b}_{\text {rs }}$ obtained from that $B \times \mathfrak{h}_{\text {reg }}$ through the isomorphism $\mu: B \times \mathfrak{h}_{\text {reg }} \stackrel{\sim}{\rightarrow} T \times \widehat{\mathfrak{b}}_{\text {rs }}$ of Proposition 4.5. Note that $\mu$ is equivariant with respect to the right action of the torus $T$ on the first component of $B \times \mathfrak{h}_{\text {reg }}$ and $T \times \widehat{\mathfrak{b}}_{\mathrm{rs}}$, respectively. Since $\dot{w}$ normalizes $T$, the birational action of $\dot{w}$ on $T \times \widehat{\mathfrak{b}}_{\mathrm{rs}}$ passes to $\widehat{\mathfrak{b}}_{\mathrm{rs}}$ through the second projection. (For $(\tau, x) \in T \times \widehat{\mathfrak{b}}_{\mathrm{rs}}$ with generic $x$, the second component of $(\tau, x) * \dot{w}$ does not depend on the choice of $\tau$ ). Hence we obtain a birational mapping $* \dot{w}: \widehat{\mathfrak{b}} \cdots \rightarrow \widehat{\mathfrak{b}}$, which gives a 
commutative diagram

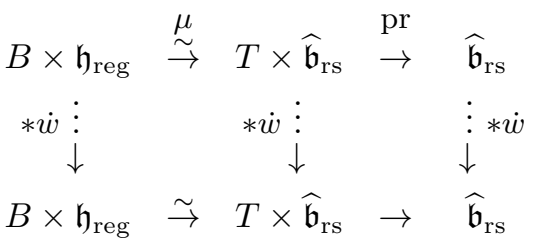

of birational mappings.

For any $b=\tau \exp (\xi) \in B$ with generic $\xi \in \widehat{\mathfrak{n}}$, let us consider the Gauss decomposition

$$
b \dot{w}=g(\dot{w}, b)^{-1}(b * \dot{w}), \quad b * \dot{w}=\widetilde{\tau} \exp (\widetilde{\xi}) \quad(\widetilde{\tau} \in T, \widetilde{\xi} \in \widehat{\mathfrak{n}}),
$$

of $b \dot{w}$. If we set $x=\operatorname{Ad}(b) h$ so that $\mu(b, h)=(\tau, x)$, then we have

$$
(\tau, x) * \dot{w}=\mu((b, h) * \dot{w})=(\widetilde{\tau}, \widetilde{x}),
$$

where

$$
\widetilde{x}=\operatorname{Ad}(b * \dot{w}) \operatorname{Ad}\left(\dot{w}^{-1}\right) h=\operatorname{Ad}(g(\dot{w}, b)) \operatorname{Ad}(b) h=\operatorname{Ad}(g(\dot{w}, b)) x
$$

since $b * \dot{w}=g(\dot{w}, b) b \dot{w}$. (As we remarked above, $\widetilde{x}$ does not depend on the $T$-component of $b$.) When $\dot{w}=\dot{s}_{i}$, in particular, from (4.127) we have

$$
g_{i}=\exp \left(\frac{-1}{\tau^{\alpha_{i}} \psi_{i}} f_{i}\right)=\exp \left(\frac{\alpha_{i}}{\varphi_{i}} f_{i}\right)=\exp \left(t_{i}(x) f_{i}\right)
$$

hence

$$
\widetilde{x}=\operatorname{Ad}\left(g_{i}\right) x=\exp \left(t_{i}(x) \operatorname{ad}\left(f_{i}\right)\right) x, \quad t_{i}=\frac{\alpha_{i}}{\varphi_{i}} .
$$

This means that the birational action $* \dot{s}_{i}: \widehat{\mathfrak{b}} \cdots \rightarrow \widehat{\mathfrak{b}}$ coincides with $\sigma_{i}$ of Proposition 4.2. On the other hand, since $\widetilde{\tau}=\tau \psi_{i}^{h_{i}}$, we also have

$$
\widetilde{\tau}_{i}=\psi_{i} \tau_{i}=-\frac{\varphi_{i}}{\alpha_{i}} \tau_{i} \tau^{-\alpha_{i}}, \quad \widetilde{\tau}_{j}=\tau_{j} \quad(j \neq i)
$$

In terms of the coordinate rings, these birational action of $\dot{w} \in \dot{W}$ on $T \times$ $\widehat{\mathfrak{b}}$ and $\widehat{\mathfrak{b}}$ induces the automorphisms $R_{\dot{w}}$ of $\mathcal{K}(\widehat{\mathfrak{b}})\left[\tau^{ \pm 1}\right]$ and $\mathcal{K}(\widehat{\mathfrak{b}})$, respectively. Combining Proposition 4.2 with (4.134) and (4.135), we obtain the following theorem.

Theorem 4.6 The birational action of $\dot{W}$ on $T \times \widehat{\mathfrak{b}}$ is determined by the algebra automorphisms $R_{\dot{s}_{i}}(i \in I)$ of the ring of Laurent polynomials $\mathcal{K}(\widehat{\mathfrak{b}})\left[\tau^{ \pm 1}\right]$ such that

(i) $\quad R_{\dot{s}_{i}}(\lambda)=\lambda-\alpha_{i}\left\langle h_{i}, \lambda\right\rangle \quad\left(i \in I, \lambda \in \mathfrak{h}^{*}\right)$

(ii) $R_{\dot{s}_{i}}\left(\tau_{j}\right)=\tau_{j} \quad(j \neq i), \quad R_{\dot{s}_{i}}\left(\tau_{i}\right)=-\frac{\varphi_{i}}{\alpha_{i}} \tau_{i} \prod_{k \in I} \tau_{k}^{-a_{k i}}$

(iii) $\quad R_{s_{i}}(\psi)=\exp \left(\frac{\lambda_{i}}{\varphi_{i}} \operatorname{ad}_{\{\}}\left(\varphi_{i}\right)\right)(\psi) \quad(i \in I, \psi \in \mathcal{O}(\widehat{\mathfrak{n}}))$,

where $\lambda_{i}=\epsilon_{i} \alpha_{i}$. 
We remark that the birational action of $\dot{W}$ on $\widehat{\mathfrak{b}}$ reduces in fact to that of the Weyl group $W=W(A)$, since the square of $* \dot{s}_{i}=\sigma_{i}$ gives the identity mapping of $\widehat{\mathfrak{b}}$ for each $i \in I$. Note, however, that $\left(* \dot{s}_{i}\right)^{2}$ is not the identity as a birational automorphism of $T \times \widehat{\mathfrak{b}}$. In fact, the action of $R_{\dot{s}_{i}}$ on the $\tau$-functions differ by the factor $-\alpha_{i}$, from that of $s_{i}$ given in Theorem 1.2.

Remark: In this section, we used for the sake of simplicity the dual lattice $L \subset \operatorname{Hom}_{\mathbb{Z}}\left(Q^{\vee}, \mathbb{Z}\right)$ of the coroot lattice $Q^{\vee}$, to specify the Cartan subgroup $T$ of $G$. A more intrinsic way would be to take an arbitrary $\mathbb{Z}$-lattice of $\mathfrak{h}^{*}$ such that $Q \subset L$ and $\left\langle h_{i}, L\right\rangle \in \mathbb{Z}$ for all $i \in I$, so that $T=\operatorname{Spec}(\mathbb{C}[L])$ and $\mathcal{O}(T)=\bigoplus_{\Lambda \in L} \mathbb{C} \tau^{\Lambda}$, where $\tau^{\Lambda}(\Lambda \in L)$ are the formal exponentials. Theorems 1.2 and 4.6 extends naturally to this setting; one has only to replace the formulas for $s_{i}\left(\tau_{j}\right)$ and $R_{s_{i}}\left(\tau_{j}\right)$ by

$$
s_{i}\left(\tau^{\Lambda}\right)=\varphi_{i}^{\left\langle h_{i}, \Lambda\right\rangle} \tau^{s_{i} . \Lambda}, \quad R_{s_{i}}\left(\tau^{\Lambda}\right)=\left(-\frac{\varphi_{i}}{\alpha_{i}}\right)^{\left\langle h_{i}, \Lambda\right\rangle} \tau^{s_{i} . \Lambda}
$$

to obtain a birational realization of $W$ and $\dot{W}$, respectively.

\section{$5 \quad$ Regularity of the $\tau$-cocycle}

In this section, we will prove that the value $\phi_{w}\left(\Lambda_{j}\right)$ of the $\tau$-cocycle is a polynomial in $\mathcal{A}=\mathcal{A}_{0}[\lambda]$ for any $w \in W$ and $j \in I$, assuming that the generalized Cartan matrix $A$ is symmetrizable. For this purpose, we may assume that the indexing set $I$ is finite, and $\mathcal{A}_{0}=\mathcal{O}(\widehat{\mathfrak{n}})$ as in the previous section. We will prove the polynomiality of $\phi_{w}\left(\Lambda_{j}\right)$ by using the action of the Kac-Moody group $G$ on the integrable highest weight $\mathfrak{g}$-modules $L(\Lambda)$ with a dominant integral weight $\Lambda \in P_{+}$. Before the proof of the regularity of the $\tau$-cocycle, we investigate the difference between the birational realization of $\dot{W}$ of Theorem 4.6 on $\mathcal{K}(\widehat{\mathfrak{b}})\left[\tau^{ \pm 1}\right]$ and that of $W$ in Theorem 1.2 .

\subsection{Comparison of the two birational realizations}

In Theorem 4.6 we gave a realization of the group $\dot{W}$ as a group of automorphisms of $\mathcal{K}(\mathfrak{b})\left[\tau^{ \pm 1}\right]$. It is also clear that each $R_{\dot{w}}$ preserves the subring $\mathcal{K}\left[\tau^{ \pm 1}\right]$ defined by the field of fractions $\mathcal{K}=Q(\mathcal{A})$ of $\mathcal{A}=\mathbb{C}[\lambda] \otimes \mathcal{A}_{0}$. On this field $\mathcal{K}$, the automorphisms $R_{\dot{s}_{i}}$ coincide with the automorphism of $s_{i}: \mathcal{K} \rightarrow \mathcal{K}$ defined by Theorem 1.1, while these two actions are different on the $\tau$-functions. We describe the difference between the two actions on the $\tau$-functions.

Note first that

$$
s_{i}\left(\tau^{\Lambda}\right)=\varphi_{i}^{\left\langle h_{i}, \Lambda\right\rangle} \tau^{s_{i} . \Lambda}, \quad R_{\dot{s}_{i}^{ \pm 1}}\left(\tau^{\Lambda}\right)=\left(\mp \frac{\varphi_{i}}{\alpha_{i}}\right)^{\left\langle h_{i}, \Lambda\right\rangle} \tau^{s_{i} . \Lambda}
$$

for each $i \in I$. This implies that $w\left(\tau^{\Lambda}\right)$ and $R_{\dot{w}}\left(\tau^{\Lambda}\right)$ differ only by a factor which is a rational function of $\alpha_{i}(i \in I)$, when $\dot{w} \in \dot{W}$ is a lift of $w \in W$. Hence, 
the ratio $N_{\dot{w}}(\Lambda)=w\left(\tau^{\Lambda}\right) / R_{\dot{w}}\left(\tau^{\Lambda}\right)$ defines a 1-cocycle of $\dot{W}$ with coefficients in $\operatorname{Hom}\left(L, \mathbb{Q}(\alpha)^{\times}\right)$:

$$
\begin{aligned}
& N_{\dot{w}}\left(\Lambda+\Lambda^{\prime}\right)=N_{\dot{w}}(\Lambda) N_{\dot{w}}\left(\Lambda^{\prime}\right) \quad\left(\Lambda, \Lambda^{\prime} \in L\right), \\
& N_{1}(\Lambda)=1, \quad N_{\dot{s}_{i}}(\Lambda)=\left(-\alpha_{i}\right)^{\left\langle h_{i}, \Lambda\right\rangle} \quad(i \in I), \\
& N_{\dot{w} \dot{w}^{\prime}}(\Lambda)=w\left(N_{\dot{w}^{\prime}}(\Lambda)\right) N_{\dot{w}}\left(w^{\prime} \cdot \Lambda\right) \quad\left(\dot{w}, \dot{w}^{\prime} \in \dot{W}\right) .
\end{aligned}
$$

When $\dot{w}=\dot{s}_{j_{1}} \cdots \dot{s}_{j_{p}}$, the factors $N_{\dot{w}}(\Lambda)(\Lambda \in L)$ are determined explicitly as

$$
N_{\dot{w}}(\Lambda)=\prod_{k=1}^{p}\left(-s_{j_{1}} \cdots s_{j_{k-1}}\left(\alpha_{j_{k}}\right)\right)^{\left\langle h_{j_{k}}, s_{j_{k+1}} \cdots s_{j_{p}} \Lambda\right\rangle} .
$$

Note that, if $w=s_{j_{1}} \cdots s_{j_{p}}$ is a reduced decomposition of $w \in W$, one has

$$
\Delta_{+} \cap w\left(\Delta_{-}\right)=\left\{s_{j_{1}} \cdots s_{j_{k-1}}\left(\alpha_{j_{k}}\right) ; k=1, \ldots, p\right\} .
$$

Assume furthermore that $\Lambda \in L$ is dominant, i.e., $\left\langle h_{i}, \Lambda\right\rangle \geq 0$ for each $i \in I$. Then one has

$$
\left\langle h_{j_{k}}, s_{j_{k+1}} \cdots s_{j_{p}} \Lambda\right\rangle \geq 0 \quad(k=1, \ldots, p) .
$$

Hence, $N_{\dot{w}}(\Lambda)$ is a polynomial in $\alpha_{i}(i \in I)$.

Proposition 5.1 Let $w=s_{j_{1}} \cdots s_{j_{p}}$ be a reduced decomposition of an element of $w \in W$ and take the corresponding element $\dot{w}=\dot{s}_{j_{1}} \cdots \dot{s}_{j_{p}}$ in $\dot{W}$. Then, for any $\Lambda \in L$, one has

$$
R_{\dot{w}}\left(\tau^{\Lambda}\right)=\frac{1}{N_{\dot{w}}(\Lambda)} w\left(\tau^{\Lambda}\right)=\frac{\phi_{w}(\Lambda)}{N_{\dot{w}}(\Lambda)} \tau^{w \cdot \Lambda},
$$

where $N_{\dot{w}}(\Lambda)$ is a rational function in the variables $\alpha_{i}(i \in I)$ with coefficients in $\mathbb{Q}$. If $\Lambda \in L$ is dominant, $N_{\dot{w}}(\Lambda)$ is a polynomial in $\alpha_{i}(i \in I)$ with integer coefficients.

\subsection{Proof of Theorem 1.3}

Recall that the $\mathbb{Z}$-module of integral weights $P$, and the cone of dominant integral weights $P_{+}$are defined by

$$
\begin{gathered}
P=\left\{\Lambda \in \mathfrak{h}^{*} ;\left\langle h_{i}, \Lambda\right\rangle \in \mathbb{Z} \quad(i \in I)\right\}, \\
P_{+}=\left\{\Lambda \in \mathfrak{h}^{*} ;\left\langle h_{i}, \Lambda\right\rangle \in \mathbb{Z}_{\geq 0}(i \in I)\right\},
\end{gathered}
$$

respectively. Note that there is a natural surjective homomorphism $P \rightarrow L$, where $L=\operatorname{Hom}_{\mathbb{Z}}\left(Q^{\vee}, \mathbb{Z}\right)$, and that, for each $\Lambda \in P$, the values $\tau^{\Lambda}$ depends only on its image in $L$. We fix a dominant integral weight $\Lambda \in P_{+}$and consider the expectation value

$$
\langle\Lambda|\cdot| \Lambda\rangle: L(\Lambda)^{\circ} \times L(\Lambda) \rightarrow \mathbb{C}
$$


at $\Lambda$. Here $L(\Lambda)$ is the integrable left $\mathfrak{g}$-module with highest weight $\Lambda$, and $L(\Lambda)^{\circ}$ is the right $\mathfrak{g}$-module with highest weight $\Lambda$, obtained from $L(\Lambda)$ by the anti-involution of $\mathfrak{g}$ such that $e_{j}^{\circ}=f_{j}, f_{j}^{\circ}=e_{j}(j \in I)$ and $h^{\circ}=h$ for $h \in \mathfrak{h}$. Note that

$$
\begin{aligned}
& \mathfrak{n}|\Lambda\rangle=0, \quad h|\Lambda\rangle=|\Lambda\rangle\langle h, \Lambda\rangle \quad(h \in \mathfrak{h}) \\
& \langle\Lambda| \mathfrak{n}_{-}=0, \quad\langle\Lambda| h=\langle h, \Lambda\rangle\langle\Lambda| \quad(h \in \mathfrak{h}) .
\end{aligned}
$$

Let $b=\tau \exp (\xi)$ be an element of $B$ with $\tau \in T, \xi \in \widehat{\mathfrak{n}}$. Then one has

$$
\langle\Lambda|b| \Lambda\rangle=\langle\Lambda|\tau \exp (\xi)| \Lambda\rangle=\langle\Lambda|\tau| \Lambda\rangle=\tau^{\Lambda} .
$$

Namely, $\tau^{\Lambda}$ is the expectation value of the Cartan component of $b \in B$ at $\Lambda$. Let $\dot{w} \in \dot{W}$ and take a generic $b \in B$ such that $b \dot{w} \in U_{-} B$. Setting $b * w=\widetilde{\tau} \exp (\widetilde{\xi})$ $(\widetilde{\tau} \in T, \widetilde{\xi} \in \widehat{\mathfrak{n}})$, we have

$$
\langle\Lambda|b * \dot{w}| \Lambda\rangle=\langle\Lambda|\widetilde{\tau} \exp (\widetilde{\xi})| \Lambda\rangle=\langle\Lambda|\widetilde{\tau}| \Lambda\rangle=\widetilde{\tau}^{\Lambda}=R_{\dot{w}}\left(\tau^{\Lambda}\right),
$$

where $R_{\dot{w}}\left(\tau^{\Lambda}\right)$ is an abbreviation for $\prod_{i \in I}\left(R_{\dot{w}} \tau_{i}\right)^{\left\langle h_{i}, \Lambda\right\rangle}$. On the other hand, we compute

$$
\langle\Lambda|b * \dot{w}| \Lambda\rangle=\langle\Lambda|g(\dot{w}, b) b \dot{w}| \Lambda\rangle=\langle\Lambda|b \dot{w}| \Lambda\rangle
$$

since $g(\dot{w}, b) \in U_{-}$. Hence we have

$$
R_{\dot{w}}\left(\tau^{\Lambda}\right)=\langle\Lambda|b \dot{w}| \Lambda\rangle .
$$

Note that $w \cdot \Lambda=\Lambda-\beta$, for some $\beta \in Q_{+}$and that there exists an element $F_{\beta} \in U\left(\mathfrak{n}_{-}\right)$of weight $-\beta$ such that $\dot{w}|\Lambda\rangle=F_{\beta}|\Lambda\rangle$. Hence we have

$$
R_{\dot{w}}\left(\tau^{\Lambda}\right)=\left\langle\Lambda\left|b F_{\beta}\right| \Lambda\right\rangle=\left\langle\Lambda\left|\tau \exp (\xi) F_{\beta}\right| \Lambda\right\rangle=\tau^{\Lambda}\left\langle\Lambda\left|\exp (\xi)_{\beta} F_{\beta}\right| \Lambda\right\rangle,
$$

where $\exp (\xi)_{\beta}$ stands for the component of weight $\beta$ of $\exp (\xi)$ :

$$
\exp (\xi)_{\beta}=\sum_{k=0}^{\infty} \frac{1}{k !} \sum_{\beta_{1}+\cdots+\beta_{k}=\beta} \xi_{\beta_{1}} \cdots \xi_{\beta_{k}} \quad \text { (finite sum). }
$$

This implies that $R_{\dot{w}}\left(\tau^{\Lambda}\right)$ is a regular function on $B$ :

$$
R_{\dot{w}}\left(\tau^{\Lambda}\right) \in \mathcal{O}(U) \tau^{\Lambda} \subset \mathcal{O}(B) .
$$

Hence, under the identification of Proposition 1.5, we have

$$
R_{\dot{w}}\left(\tau^{\Lambda}\right) \in \mathcal{O}\left(\widehat{\mathfrak{b}}_{\mathrm{rs}}\right)\left[\tau^{ \pm 1}\right]
$$

Since we already know that the left hand side belongs to $\mathcal{K}(\widehat{\mathfrak{b}}) \tau^{w \cdot \Lambda}$, we obtain

$$
R_{\dot{w}}\left(\tau^{\Lambda}\right) \in \mathcal{O}\left(\widehat{\mathfrak{b}}_{\mathrm{rs}}\right) \tau^{w \cdot \Lambda}
$$

namely,

$$
\frac{\phi_{w}(\Lambda)}{N_{\dot{w}}(\Lambda)} \in \mathcal{O}(\widehat{\mathfrak{b}})\left[\alpha^{-1}\left(\alpha \in \Delta_{+}\right)\right]
$$


Let $w=s_{j_{1}} \cdots s_{j_{p}}$ be a reduced decomposition of an element $w \in W$ and set $\dot{w}=\dot{s}_{j_{1}} \cdots \dot{s}_{j_{p}}$. In this setting, the normalization factor $N_{\dot{w}}(\Lambda)$ is a polynomial in $\alpha_{i}(i \in I)$. Hence, we have

$$
\phi_{w}(\Lambda) \in \mathcal{O}(\widehat{\mathfrak{b}})\left[\alpha^{-1}\left(\alpha \in \Delta_{+}\right)\right] \subset \mathbb{C}(\alpha) \otimes \mathcal{O}(\widehat{\mathfrak{n}})=\mathbb{C}(\alpha) \otimes \mathcal{A}_{0} .
$$

where $\mathcal{A}_{0}=\mathcal{O}(\widehat{\mathfrak{n}})=\operatorname{Sym}\left(\mathfrak{n}^{*}\right)$.

We now prove that

$$
\phi_{w}(\Lambda) \in \mathcal{A}=\mathbb{C}[\alpha] \otimes \mathcal{A}_{0} \quad(w \in W),
$$

by the induction on the length $\ell(w)=p$. Set $w^{\prime}=s_{j_{2}} \cdots s_{j_{p}}$ so that $w=s_{j_{1}} w^{\prime}$. Then, by the cocycle property of $\phi$, we have

$$
\phi_{w}(\Lambda)=s_{j_{1}}\left(\phi_{w^{\prime}}(\Lambda)\right) \phi_{s_{j_{1}}}\left(w^{\prime} \Lambda\right)=s_{j_{1}}\left(\phi_{w^{\prime}}(\Lambda)\right) \varphi_{j_{1}}^{\left\langle h_{j_{1}}, w^{\prime} \Lambda\right\rangle} .
$$

By the induction hypothesis, we have $\phi_{w^{\prime}}(\Lambda) \in \mathcal{A}$. Since $s_{j_{1}}(\mathcal{A}) \subset \mathcal{A}\left[\varphi_{j_{1}}^{-1}\right]$, we see $\phi_{w}(\Lambda) \in \mathcal{A}\left[\varphi_{j_{1}}^{-1}\right]$. On the other hand, we already know that $\phi_{w}(\Lambda) \in$ $\mathbb{C}(\alpha) \otimes \mathcal{A}_{0}$. Since $\mathcal{A}_{0}=\operatorname{Sym}\left(\mathfrak{n}^{*}\right)$ is a polynomial ring including $\varphi_{j_{1}}$ as an indeterminate, we have

$$
\mathbb{C}[\alpha] \otimes \mathcal{A}_{0}\left[\varphi_{j_{1}}^{-1}\right] \cap \mathbb{C}(\alpha) \otimes \mathcal{A}_{0}=\mathbb{C}[\alpha] \otimes \mathcal{A}_{0}=\mathcal{A} .
$$

Hence $\phi_{w}(\Lambda) \in \mathcal{A}$, as desired. This completes the proof of Theorem 1.3.

Remark: When the Poisson algebra $\mathcal{A}_{0}=\operatorname{Sym}\left(\mathfrak{n}^{*}\right)$ has a $\mathbb{Z}$-form, one can show furthermore that each $\phi_{w}\left(\Lambda_{j}\right)$ is defined over $\mathbb{Z}$. To be more precise, suppose that there exists a $\mathbb{Z}$-submodule $\mathcal{S} \subset \mathfrak{n}^{*}$ such that

(i) $\quad\{\mathcal{S}, \mathcal{S}\} \subset \mathcal{S}$, and $\mathcal{S} \otimes_{\mathbb{Z}} \mathbb{C}=\mathfrak{n}^{*}$.

(ii) $\varphi_{j} \in \mathcal{S}$ for any $j \in I$.

(iii) $\frac{1}{k !} \operatorname{ad}_{\{\}}\left(\varphi_{j}\right)^{k}(\mathcal{S}) \subset \mathcal{S}$ for any $j \in I$ and $k=0,1,2, \ldots$

Then, for any dominant integral weight $\Lambda \in P_{+}$, one has $\phi_{w}(\Lambda) \in \mathbb{Z}[\lambda][\mathcal{S}]$ for all $w \in W$. Namely, each $\phi_{w}(\Lambda)$ is expressed as a linear combination of products of $\lambda_{i}=\epsilon_{i} \alpha_{i}(i \in I)$ and elements of $\mathcal{S}$, with integer coefficients.

\section{Concluding remarks}

In this paper, we proposed a general method to realize an arbitrary Weyl group $W=W(A)$ as a group of automorphisms of the field of rational functions $\mathcal{K}=$ $\mathbb{C}\left(\lambda_{i}, \varphi_{i},\left\{\varphi_{i}, \varphi_{j}\right\}, \cdots\right)$, starting from a nilpotent Poisson algebra generated by $\varphi_{i}(i \in I)$ with the Serre relations. Also we discussed how this realization arise from the Weyl group of the Kac-Moody group through the Gauss decomposition.

Here we give two remarks about the cases when the GCM is of affine type.

(1) When the GCM is of affine type, our construction already provides a class of discrete integrable systems associated with the affine root system, as is discussed 
in our previous paper [9]. They can be regarded as a universal version containing higher $\varphi$-variables (or $f$-variables [9]).

(2) Birational realizations of affine Weyl groups, in the sense of this paper, arise also as groups of Bäcklund transformations for nonlinear differential equations of Painlevé type, obtained by certain reduction from the Drinfeld-Sokolov hierarchy of modified type 四.

Detail of such specific features of the affine cases will be discussed in our forthcoming paper.

\section{Acknowledgments}

The authors are grateful to Professors T. Tanisaki, Y. Saito and K. Iohara for valuable discussions. A part of this paper was prepared during the stay of one of the authors (M.N.) at the Erwin Schrödinger International Institute for Mathematical Physics, Vienna, for the research program on Representation Theory. He would like to express his thanks to Professors V.G. Kac and A.A. Kirillov, for their hospitality during his stay at ESI.

\section{References}

[1] V.G. Drinfeld and V.V. Sokolov: Lie algebras and equations of Korteweg-de Vries type, Sov. J. Math. 30(1985), 1975-2036.

[2] S. Fomin and A.N. Kirillov: Universal exponential solution of the YangBaxter equation, Lett. Math. Phys. 37(1996), 273-284.

[3] V.G. Kac: Infinite dimensional Lie algebras, Third edition, Cambridge University Press, 1990.

[4] V.G. Kac and D.H. Peterson: Regular functions on certain infinite dimensional groups, in Arithmetic and Geometry (Eds. M. Artin and J. Tate), Progress in Math. 36, Birkhäuser, Boston, 1983, 141-166.

[5] M. Kashiwara: The flag manifold of Kac-Moody Lie algebras, in "Algebraic Analysis, Geometry, and Number Theory" (Baltimore, MD, 1988), Johns Hopkins University Press, Baltimore MD, 1989, 161-190.

[6] M. Kashiwara and T. Tanisaki: Kazhdan-Lusztig conjecture for affine Lie algebras with negative level, Duke Math. J. 77(1995), 21-62.

[7] O. Mathieu: Formules de caractères pour les algèbres de Kac-Moody générales, Astérisque 159-160, 1988.

[8] M. Noumi, S. Okada, K. Okamoto and H. Umemura: Special polynomials associated with the Painlevé equations. II, in Integrable Systems and Algebraic Geometry, Proceedings of the Taniguchi Symposium 1997 (Eds. 
M.-H. Saito, Y. Shimizu and K. Ueno), World Sci. Publishing, River Edge, NJ, 1998, 349-372.

[9] M. Noumi and Y. Yamada: Affine Weyl groups, discrete dynamical systems and Painlevé equations, Comm. Math. Phys. 199(1998), 281-295.

[10] P. Slodowy: On the geometry of Schubert varieties attached to KacMoody Lie algebras, Canadian Mathematical Society Conference Proceedings 6(1986), 405-442.

[11] J. Tits: Définition par générateurs et relations de groupes avec $B N$-paires, C. R. Acad. Sci. Paris 293(1981), 317-322. 